IMA Journal of Numerical Analysis (2005) 25, 379-407

doi:10.1093/imanum/drh017

Advance Access publication 21 January 2005

\title{
Finite elements on degenerate meshes: inverse-type inequalities and applications
}

\author{
I. G. GRAHAM $\dagger$ \\ Department of Mathematical Sciences, University of Bath, Bath BA2 7AY, UK \\ W. HACKBUSCH \\ Max-Planck-Institut Mathematik in den Naturwissenschaften, D-04103 Leipzig, \\ Inselstr. 22-26, Germany
}

AND

S. A. SAUTER

Institut für Mathematik, Universität Zürich, Winterthurerstr 190, CH-8057 Zürich, Switzerland

[Received on 27 November 2002; revised on 11 May 2004]

\begin{abstract}
In this paper we obtain a range of inverse-type inequalities which are applicable to finite-element functions on general classes of meshes, including degenerate meshes obtained by anisotropic refinement. These are obtained for Sobolev norms of positive, zero and negative order. In contrast to classical inverse estimates, negative powers of the minimum mesh diameter are avoided. We give two applications of these estimates in the context of boundary elements: (i) to the analysis of quadrature error in discrete Galerkin methods and (ii) to the analysis of the panel clustering algorithm. Our results show that degeneracy in the meshes yields no degradation in the approximation properties of these methods.

Keywords: finite elements; degenerate meshes; boundary element method; quadrature; panel-clustering.
\end{abstract}

\section{Introduction}

For $d=2$ or 3 , let $\Omega \subset \mathbb{R}^{3}$ denote either a bounded domain $(d=3)$ or a bounded surface with or without boundary $(d=2)$. Suppose that $\Omega$ is decomposed into a mesh of tetrahedra/bricks $(d=3)$ or curvilinear triangles/quadrilaterals $(d=2)$. Then classical inverse estimates give

$$
\|u\|_{H^{s}(\Omega)} \lesssim h_{\min }^{-s}\|u\|_{L^{2}(\Omega)} \lesssim h_{\min }^{-2 s}\|u\|_{H^{-s}(\Omega)},
$$

for a suitable range of positive $s$ and for all functions $u \in H^{s}(\Omega)$ which are piecewise polynomials of degree $\leqslant m$ with respect to this mesh. (Here the notation $A \lesssim B$ means that $A / B$ is bounded by a constant independent of the mesh and independent of $u$ - for a more precise statement, see Section 2.) The quantity $h_{\min }$ is the minimum diameter of all the elements of the mesh and (1.1) holds under the assumption of shape regularity, i.e. $\rho_{\tau} \gtrsim h_{\tau}$ for each $\tau$, where $h_{\tau}$ is the diameter of $\tau$ and $\rho_{\tau}$ is the diameter of the largest inscribed sphere (see Definition 2.1). Such estimates are regularly used in finiteelement analysis. When the mesh is quasiuniform $\left(h \lesssim h_{\min }\right.$, where $h$ is the maximum diameter of all the elements), they can be used to obtain convergence rates in powers of $h$ for various quantities in various norms. However, practical meshes are often non-quasiuniform and then the negative powers of $h_{\min }$ in (1.1) may give rise to overly pessimistic convergence rates. In Dahmen et al. (2004), less pessimistic

\footnotetext{
$\dagger^{\dagger}$ Email: igg@maths.bath.ac.uk
} 
replacements for (1.1) have been derived, a particular case being

$$
\|u\|_{H^{s}(\Omega)} \lesssim\left\|h^{-s} u\right\|_{L^{2}(\Omega)} \lesssim\left\|h^{-2 s} u\right\|_{H^{-s}(\Omega)},
$$

where $h: \Omega \rightarrow \mathbb{R}$ is now a continuous piecewise linear mesh function whose value on each element $\tau$ reflects the diameter of that element (i.e. $\left.h_{\tau} \lesssim h\right|_{\tau} \lesssim h_{\tau}$ ).

Estimates (1.2) have several applications, e.g. to the analysis of quadrature errors in discrete Galerkin boundary element methods (Graham et al., 2000a) and to the analysis of the mortar element method (Dahmen et al., 2004). In fact, Dahmen et al. (2004) contains more general versions of (1.2), e.g. in the Sobolev space $W^{s, p}(\Omega)$ and in related Besov spaces. While the left-hand inequality in (1.2) is wellknown, at least in the Sobolev space case, the right-hand inequality requires rather delicate analysis.

In this paper we obtain more general versions of (1.2) which do not require the mesh sequence to be shape-regular. A typical estimate is

$$
\|u\|_{H^{s}(\Omega)} \lesssim\left\|\rho^{-s} u\right\|_{L^{2}(\Omega)} \lesssim\left\|\rho^{-2 s} u\right\|_{H^{-s}(\Omega)},
$$

where the mesh function $\rho: \Omega \rightarrow \mathbb{R}$ is now a continuous piecewise linear function whose value on each element $\tau$ reflects the diameter of the largest inscribed sphere, introduced in Definition 2.1. Estimates (1.3) hold under the rather weak assumptions that (i) the quantities $h_{\tau}$ and $\rho_{\tau}$ are locally quasiuniform (i.e. $h_{\tau} / h_{\tau^{\prime}} \lesssim 1$ and $\rho_{\tau} / \rho_{\tau^{\prime}} \lesssim 1$ for all neighbouring elements $\tau, \tau^{\prime}$ ) and (ii) the number of elements which touch any element remains bounded as the mesh is refined (see Assumption 2.6). These assumptions admit degenerate meshes, containing long thin 'stretched' elements, which are typically used for approximating edge singularities or boundary layers in solutions of PDEs. Our estimates (1.3) hold true when all the elements $\tau$ of a mesh are obtained by suitable maps from a single unit element, as is usual for finite-element spaces. For the purpose of a readable introduction we have here written our estimates (1.3) in a very compact form. In fact, the range of $s$ for which the right-hand inequality in (1.3) holds may be greater then that for which the left-hand inequality holds and we shall give precise ranges in Section 3.

It is expected that these estimates will have a range of applications similar to those already identified above for (1.2). In particular, we already used a special case of (1.3) to analyse quadrature errors for a Galerkin boundary element discretization of a model screen problem in Graham et al. (1999). In this paper we give as applications a more general Galerkin quadrature error analysis, as well as an error analysis of the panel clustering algorithm in the presence of degenerate meshes.

Our inverse estimates are proved in Section 3. We briefly introduce the well-known Galerkin boundary element method in Section 4. The analysis of Galerkin quadrature is given in Section 5. Quadrature almost always has to be employed in practical computations; a general analysis for shaperegular meshes was included in Graham et al. (2000a). In Section 5, with the help of (1.3), we generalize the results of Graham et al. (2000a) to degenerate meshes. The results turn out to be qualitatively the same as those in Graham et al. (2000a): in the far field the degeneracy of the mesh has no effect on the required precision of the quadrature needed to preserve the accuracy of the Galerkin method. The error analysis of the panel clustering algorithm is given in Section 6. This algorithm (Hackbusch \& Nowak, 1989; Sauter, 1997) provides an alternative representation of the finite-dimensional Galerkin operator which has the same order of accuracy as the standard representation. The multiplication of the panel clustering representation with any vector has complexity $\mathcal{O}\left(N \log ^{\kappa} N\right)$, for some (small) $\kappa$, where $N$ is the number of degrees of freedom. This should be compared with the complexity $\mathcal{O}\left(N^{2}\right)$ of the standard matrix representation. Until now the accuracy and complexity analysis for this algorithm was obtained only for quasiuniform meshes. In Section 6 we extend the accuracy analysis to the case of much more 
general (including degenerate) meshes using (1.3). Again we find the error estimate is qualitatively the same as in the quasiuniform case.

It turns out, however, that when the conventional panel clustering algorithm is applied in practice to some discretizations on degenerate mesh sequences, it has a complexity higher than the $\mathcal{O}\left(N \log ^{\kappa} N\right)$ mentioned above. In a subsequent paper (Graham et al., in preparation) we shall elaborate on this and we shall propose a new variant of the panel clustering algorithm which is optimal for this type of mesh. The results here, depending on (1.3), are crucial for the analysis which will be given in Graham et al. (in preparation).

\section{Meshes and finite elements}

Throughout Sections 2 and 3, $\Omega$ will denote a bounded $d$-dimensional subset of $\mathbb{R}^{3}$, for $d=2$ or 3 . More precisely, when $d=3, \Omega$ will denote a bounded domain in $\mathbb{R}^{3}$ and for $d=2, \Omega$ will denote a bounded two-dimensional piecewise smooth Lipschitz manifold in $\mathbb{R}^{3}$ which may or may not have a boundary. The case when $\Omega$ is a bounded domain in $\mathbb{R}^{2}$ is then included as a special case, by trivially embedding it into $\mathbb{R}^{3}$.

We define the Sobolev space $H^{s}(\Omega), s \geqslant 0$, in the usual way (see e.g. Hackbusch, 1992 or McLean, 2000). Note that in the case $d=2$ the range of $s$ for which $H^{s}(\Omega)$ is defined may be limited, depending on the global smoothness of the surface $\Omega$. Throughout, we let $[-k, k]$ denote the range of Sobolev indices for which we are going to prove the inverse estimates (where $k$ is a positive integer), and we assume that $H^{s}(\Omega)$ is defined for all $s \in[-k, k]$, and that $H^{-s}(\Omega)$ is the dual of $H^{s}(\Omega)$, for $s>0$. (For example, when $d=2$ and $\Omega$ is a Lipschitz manifold, then $-1 \leqslant s \leqslant 1$.) We assume that $\Omega$ is decomposed into a mesh $\mathcal{T}$ of relatively open pairwise-disjoint finite elements $\tau \subset \Omega$ with the property $\bar{\Omega}=\cup\{\bar{\tau}: \tau \in \mathcal{T}\}$.

In our applications to boundary integral equations in Sections 4-6, we will restrict to equations posed on closed bounded surfaces in $\mathbb{R}^{3}$. Thus we avoid dealing explicitly with special subspaces of $H^{s}(\Omega)$ which vanish on the boundary (for $s \geqslant 0$ ), and their dual spaces. However, we note that the inverse estimates which we shall obtain for functions in $H^{s}(\Omega)$ obviously hold also for functions in any subspace of $H^{s}(\Omega)$.

Definition 2.1 (Mesh parameters) For each $\tau \in \mathcal{T},|\tau|$ denotes its $d$-dimensional measure, $h_{\tau}$ denotes its diameter and $\rho_{\tau}$ is the diameter of the largest sphere centred at a point in $\tau$ whose intersection with $\bar{\Omega}$ lies entirely inside $\bar{\tau}$. For any other simplex or cube $t \in \mathbb{R}^{d}$ (not necessarily an element of $\mathcal{T}$ ) we define $h_{t}$ and $\rho_{t}$ in the same way.

In order to impose a simple geometric character on the mesh $\tau$, we assume that each $\tau \in$ $\mathcal{T}$ is diffeomorphic to a simple unit element. More precisely, let $\hat{\sigma}^{3}$ denote the unit simplex with vertices $(0,0,0),(1,0,0),(0,1,0),(0,0,1)$, let $\hat{\kappa}^{3}$ denote the unit cube with vertices $\{(x, y, z): x, y, z \in\{0,1\}\}$. In two dimensions, define $\hat{\sigma}^{2}$ to be the unit simplex with vertices $(0,0),(1,0)$ and $(0,1)$ and define $\hat{\kappa}^{2}$ to be the unit square with vertices $\{(x, y): x, y \in\{0,1\}\}$.

Then we assume that for each $\tau \in \mathcal{T}$, there exists a unit element $\hat{\tau}=\hat{\sigma}^{d}$ or $\hat{\kappa}^{d}$ and a bijective map $\chi_{\tau}: \hat{\tau} \rightarrow \tau$, with $\chi_{\tau}$ and $\chi_{\tau}^{-1}$ both smooth. (Here, for simplicity, 'smooth' means $\mathcal{C}^{\infty}$.) We also let $|\hat{\tau}|$ denote the $d$-dimensional measure of $\hat{\tau}$ and $h_{\hat{\tau}}$ denote its diameter. Since $\chi_{\tau}$ is smooth, each element $\tau \in \mathcal{T}$ is either a curvilinear tetrahedron/brick $(d=3)$ or a curvilinear triangle/rectangle $(d=2)$. The mesh $\mathcal{T}$ is allowed to contain both types of elements. Each element has vertices and edges (defined to be the images of the vertices and edges of the corresponding unit element under $\chi_{\tau}$ ). In the 3D case the element also has faces, comprising the images of the faces of the unit element. For a suitable index set 
$\mathcal{N}$, we let $\left\{\mathbf{x}_{p}: p \in \mathcal{N}\right\}$ denote the set of all vertices of $\mathcal{T}$. We assume the mesh is conforming, i.e. for each $\tau, \tau^{\prime} \in \mathcal{T}$ with $\tau \neq \tau^{\prime}, \bar{\tau} \cap \overline{\tau^{\prime}}$ is allowed to be either empty, a node, an edge or (when $d=3$ ) a face of both $\tau$ and $\tau^{\prime}$. The requirement that $\chi_{\tau}$ is smooth ensures that edges of $\Omega(d=2)$ and edges of $\partial \Omega$ $(d=3)$ are confined to edges of elements $\tau \in \mathcal{T}$. Let $J_{\tau}$ denote the $3 \times d$ Jacobian of $\chi_{\tau}$. Then

$$
g_{\tau}:=\left\{\operatorname{det} J_{\tau}^{T} J_{\tau}\right\}^{1 / 2}
$$

is the Gram determinant of the map $\chi_{\tau}$, which appears in the change of variable formula: $\int_{\tau} f(\mathbf{x}) \mathrm{d} x=$ $\int_{\hat{\tau}} f\left(\chi_{\tau}(\hat{\mathbf{x}})\right) g_{\tau}(\hat{\mathbf{x}}) \mathrm{d} \hat{x}$. To ensure that the map $\chi_{\tau}$ is sufficiently regular we shall make the following assumptions on $J_{\tau}$.

AsSUMPTION 2.2 (Mapping properties)

$$
\begin{aligned}
D^{-1}|\tau|^{2} & \leqslant \operatorname{det}\left\{J_{\tau}(\hat{\mathbf{x}})^{T} J_{\tau}(\hat{\mathbf{x}})\right\} \leqslant D|\tau|^{2}, \\
E \rho_{\tau}^{2} & \leqslant \lambda_{\min }\left\{J_{\tau}(\hat{\mathbf{x}})^{T} J_{\tau}(\hat{\mathbf{x}})\right\}
\end{aligned}
$$

uniformly in $\hat{\mathbf{x}} \in \hat{\tau}$, for all $\tau \in \mathcal{T}$, with positive constants $D, E$ independent of $\tau$.

(Throughout this section, for a symmetric matrix $A, \lambda_{\min }(A)$ and $\lambda_{\max }(A)$ denote respectively the minimum and maximum eigenvalues of $A$.) Assumption 2.2 is satisfied in a number of standard cases.

EXAMPLE 2.3 Suppose either $d=2$ and $\tau \subset \Omega$, where $\Omega$ is a planar polygon (assumed without loss of generality to lie in the plane $x_{3}=0$ ) or $d=3$. Suppose also that $\chi_{\tau}$ is an affine map. Then the Jacobian $J_{\tau}$ can be identified with a $d \times d$ constant matrix and it is well known (e.g. Ciarlet, 1978) that $\operatorname{det} J_{\tau}=|\tau| /|\hat{\tau}|$ and that $\left\|J_{\tau}^{-1}\right\|_{2} \leqslant h_{\hat{\tau}} \rho_{\tau}^{-1}$, from which the estimates (2.1a,b) follow.

Proceeding to the case when $\Omega$ is a surface we give the following example.

EXAMPLE 2.4 Suppose $d=2$ and let $\Omega$ be the surface of a polyhedron. Let $\tau$ be a triangle with vertices $\mathbf{x}_{1}, \mathbf{x}_{2}, \mathbf{x}_{3} \in \mathbb{R}^{3}$ and choose $\chi_{\tau}$ to be the affine map $\chi_{\tau}(\hat{\mathbf{x}})=\mathbf{x}_{1}+\hat{x}_{1} \mathbf{a}+\hat{x}_{2} \mathbf{b}$, where $\mathbf{a}=\mathbf{x}_{2}-\mathbf{x}_{1}$, $\mathbf{b}=\mathbf{x}_{3}-\mathbf{x}_{1}$. Then

$$
J_{\tau}^{T} J_{\tau}=\left[\begin{array}{ll}
|\mathbf{a}|^{2} & \mathbf{a}^{T} \mathbf{b} \\
\mathbf{a}^{T} \mathbf{b} & |\mathbf{b}|^{2}
\end{array}\right], \operatorname{det} J_{\tau}^{T} J_{\tau}=|\mathbf{a} \times \mathbf{b}|^{2}=4|\tau|^{2},
$$

from which (2.1a) follows. If we denote the eigenvalues of $J_{\tau}^{T} J_{\tau}$ by $0<\lambda_{-}<\lambda_{+}$, then we can easily obtain the relations $\lambda_{+} \leqslant \lambda_{-}+\lambda_{+}=|\mathbf{a}|^{2}+|\mathbf{b}|^{2} \leqslant 2 h_{\tau}^{2}$ and $\lambda_{-} \lambda_{+}=4|\tau|^{2}$ which imply (2.1b).

Finite elements on curved surfaces can similarly be shown to satisfy Assumption 2.2, for example when the map $\chi_{\tau}$ is sufficiently close to affine.

In many applications quadrilateral or hexahedral elements are important. Consider, for example, quadrilateral elements $\tau$ obtained by mapping from the unit element $\hat{\kappa}^{2}=(0,1)^{2}$. If the map is affine, then the estimates for (2.1a,b) obtained in Example 2.4 carry over verbatim. However, only parallelograms can be obtained by applying affine maps to $\hat{\kappa}^{2}$. More general quadrilaterals can be obtained using bilinear maps and it turns out that, under quite moderate assumptions, $(2.1 \mathrm{a}, \mathrm{b})$ still hold. Since there is no obvious reference for this result we state and prove it here as a proposition.

Consider any quadrilateral element $\tau$. A parallelogram $\pi$ (considered as an open subset of $\mathbb{R}^{2}$ ) will be called an inscribed parallelogram for $\tau$ if $\pi \subset \tau$ and if two adjacent edges of $\pi$ are identical with two adjacent edges of $\tau$. It is easy to show that any convex quadrilateral $\tau$ has at least one inscribed parallelogram. 


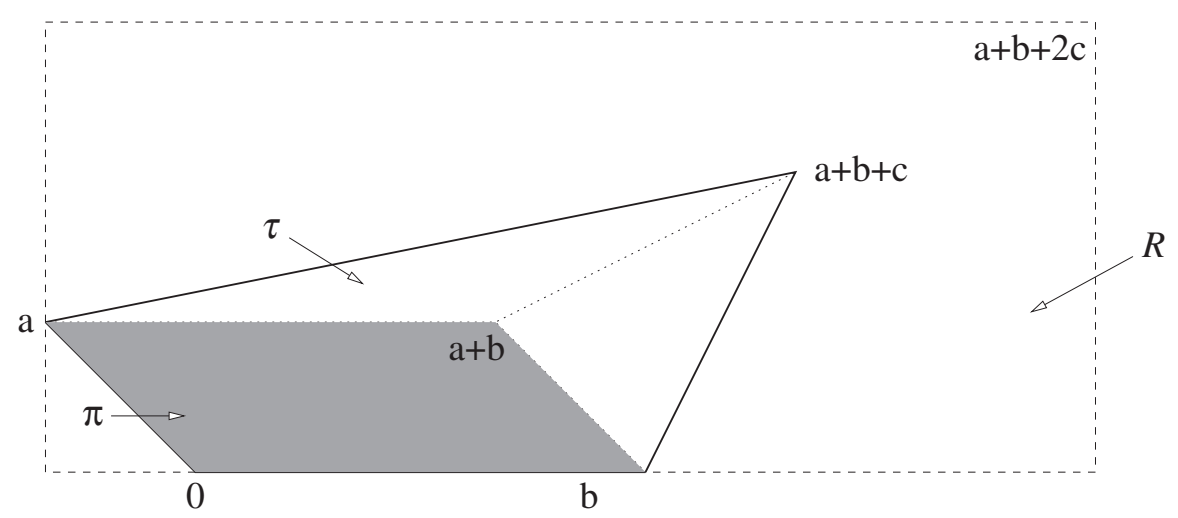

FIG. 1. Quadrilateral $\tau$ (with $\mathbf{x}_{0}:=\mathbf{0}$ ), parallelogram $\pi$ and bounding rectangle $R$.

PROPOSITION 2.5 Let $\tau$ be a convex planar quadrilateral obtained by applying a bilinear mapping to $\hat{\kappa}^{2}$. Let $\pi$ be any inscribed parallelogram for $\tau$. Then the estimates $(2.1 \mathrm{a}, \mathrm{b})$ hold uniformly in $\hat{\mathbf{x}} \in \hat{\tau}$ with $D, E$ depending continuously on the ratio $r:=|\pi| /|\tau| \in(0,1]$.

REMARK It follows that if the ratio $|\pi| /|\tau|$ is bounded below by some constant $\gamma>0$ say, for all elements $\tau$ as the mesh is refined, then (2.1a,b) hold (with $D$ and $E$ dependent on $\gamma$ ). There are obvious degenerate elements which satisfy this, for example any parallelograms (no matter how small the smallest interior angles are) satisfy it. Similarly, 'moderately' distorted parallelograms also satisfy it.

Proof. We assume that $\tau$ has vertices $\mathbf{x}_{0}, \mathbf{x}_{0}+\mathbf{a}, \mathbf{x}_{0}+\mathbf{b}, \mathbf{x}_{0}+\mathbf{a}+\mathbf{b}+\mathbf{c}$ and that the inscribed parallelogram $\pi$ has vertices $\mathbf{x}_{0}, \mathbf{x}_{0}+\mathbf{a}, \mathbf{x}_{0}+\mathbf{b}, \mathbf{x}_{0}+\mathbf{a}+\mathbf{b}$. Without loss of generality, we may assume that $|\mathbf{a}| \leqslant|\mathbf{b}|$ (see Fig. 1 in which we put $\mathbf{x}_{0}$ at $\mathbf{0}$ for convenience).

For this proof only, we introduce the notation $A \precsim B$ to mean that $\mathrm{A} / \mathrm{B}$ is bounded above by a constant which depends continuously on $r \in(0,1]$, and we attach the obvious analogous meanings to $\succsim$ and $\simeq$.

(a) Elementary trigonometry shows that $|\mathbf{c}| \precsim|\mathbf{b}|$ and $\left|P^{\perp} \mathbf{c}\right| \precsim\left|P^{\perp} \mathbf{a}\right|$, where $P^{\perp}$ denotes the projection orthogonal to $\mathbf{b}$.

(b) Now let $R$ be the smallest rectangle containing $\pi, \tau$, and the shifted vertices $\left\{\mathbf{x}_{0}+\mathbf{a}+\mathbf{c}, \mathbf{x}_{0}+\right.$ $\left.\mathbf{b}+\mathbf{c}, \mathbf{x}_{0}+\mathbf{a}+\mathbf{b}+2 \mathbf{c}\right\}$ (see Fig. 1). The length of $R$ is bounded by $\precsim|\mathbf{b}|+2|\mathbf{c}| \precsim|\mathbf{b}|$ and the height of $R$ is bounded by $\precsim\left|P^{\perp} \mathbf{a}\right|+2\left|P^{\perp} \mathbf{c}\right| \precsim\left|P^{\perp} \mathbf{a}\right|$, with both estimates making use of part (a). Hence we have

$$
|\tau| \simeq|\pi|=|\mathbf{b}|\left|P^{\perp} \mathbf{a}\right| \simeq|R| .
$$

(c) The bilinear mapping from $\hat{\kappa}^{2}$ to $\tau$ may be written $\chi_{\tau}(\hat{\mathbf{x}})=\mathbf{x}_{0}+\hat{x}_{1} \mathbf{a}+\hat{x}_{2} \mathbf{b}+\hat{x}_{1} \hat{x}_{2} \mathbf{c}$. With $J_{\tau}(\hat{\mathbf{x}})$ denoting its Jacobian, it can easily be seen that $J_{\tau}^{T}(\hat{\mathbf{x}}) J_{\tau}(\hat{\mathbf{x}})$ has the form (2.2) with $\mathbf{a}, \mathbf{b}$ replaced by $\mathbf{a}^{\prime}(\hat{\mathbf{x}}):=\mathbf{a}+\hat{x}_{2} \mathbf{c}, \mathbf{b}^{\prime}(\hat{\mathbf{x}}):=\mathbf{b}+\hat{x}_{1} \mathbf{c}$. Hence, $\operatorname{det} J_{\tau}^{T}(\hat{\mathbf{x}}) J_{\tau}(\hat{\mathbf{x}})=4|\pi(\hat{\mathbf{x}})|^{2}$, where $\pi(\hat{\mathbf{x}})$ is the parallelogram with vertices $\mathbf{x}_{0}, \mathbf{x}_{0}+\mathbf{a}^{\prime}(\hat{\mathbf{x}}), \mathbf{x}_{0}+\mathbf{b}^{\prime}(\hat{\mathbf{x}}), \mathbf{x}_{0}+\mathbf{a}^{\prime}(\hat{\mathbf{x}})+\mathbf{b}^{\prime}(\hat{\mathbf{x}})$. Since $\pi(\hat{\mathbf{x}}) \subset R$ for all $0 \leqslant \hat{x}_{1}, \hat{x}_{2} \leqslant 1$, we can employ part (b) to obtain $\left(\operatorname{det} J_{\tau}^{T} J_{\tau}\right)(\hat{\mathbf{x}}) \leqslant 4 R^{2} \precsim 4|\tau|^{2}$, and the right-hand inequality in (2.1a) follows. 


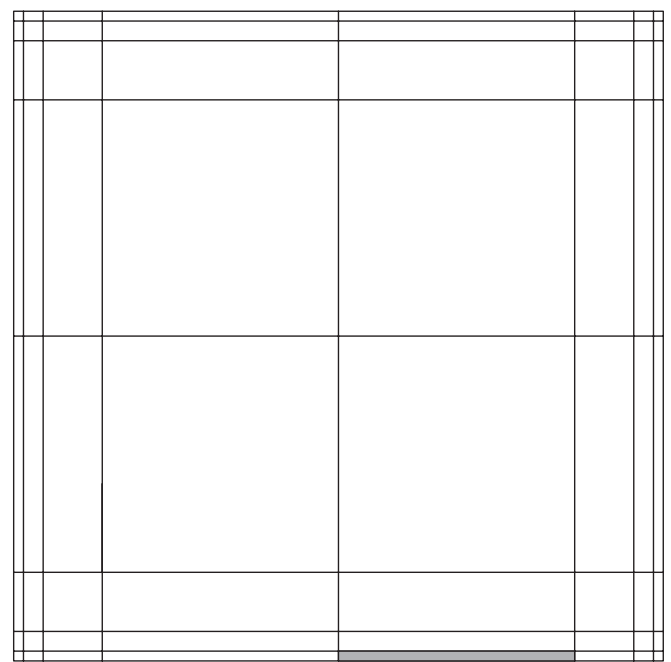

FIG. 2. Illustration of a graded mesh. Some elements become very long and thin as e.g. the shaded one in the figure. We have chosen here a smaller grading exponent $g<5$ for illustration purpose only.

To obtain the left-hand inequality in (2.1a), let (p, q) denote the matrix with columns $\mathbf{p}$ and $\mathbf{q}$. Then note (from Fig. 1) that $\mathbf{a}, \mathbf{b}, \mathbf{c}$ satisfy $\operatorname{det}(\mathbf{c}, \mathbf{a}) \geqslant 0$ and $\operatorname{det}(\mathbf{b}, \mathbf{c}) \geqslant 0$. Hence, $|\pi(\hat{\mathbf{x}})|=$ $\operatorname{det}\left(\mathbf{b}^{\prime}(\hat{\mathbf{x}}), \mathbf{a}^{\prime}(\hat{\mathbf{x}})\right)=\operatorname{det}(\mathbf{b}, \mathbf{a})+\hat{x}_{1} \operatorname{det}(\mathbf{b}, \mathbf{c})+\hat{x}_{2} \operatorname{det}(\mathbf{c}, \mathbf{a})+\hat{x}_{1} \hat{x}_{2} \operatorname{det}(\mathbf{c}, \mathbf{c}) \geqslant \operatorname{det}(\mathbf{b}, \mathbf{a})=|\pi| \succsim|\tau|$. The proof of (2.1b) is analogous to the proof of (2.1b) in Example 2.4.

Assumption 2.2 describes the quality of the maps which take the unit element $\hat{\tau}$ to each $\tau$. We also need assumptions on how the size and shape of neighbouring elements in our mesh may vary. Here we impose only very weak local conditions which require the meshes to be neither quasi-uniform nor shape-regular. Throughout the rest of this paper we make the following assumption.

Assumption 2.6 (Mesh properties) For some $K, L \in \mathbb{R}^{+}$and $M \in \mathbb{N}$, we assume that, for all $\tau, \tau^{\prime} \in$ $\mathcal{T}$ with $\bar{\tau} \cap \overline{\tau^{\prime}} \neq \emptyset$,

$$
\begin{aligned}
& h_{\tau} \leqslant K h_{\tau^{\prime}}, \quad \rho_{\tau} \leqslant L \rho_{\tau^{\prime}}, \\
& \max _{p \in \mathcal{N}} \#\left\{\tau \in \mathcal{T}: \mathbf{x}_{p} \in \bar{\tau}\right\} \leqslant M .
\end{aligned}
$$

Note that condition (2.3a) requires that $h_{\tau}$ and $\rho_{\tau}$ do not vary too rapidly between neighbouring elements. This allows elements with large aspect ratio, provided their immediate neighbours have a comparable aspect ratio.

EXAMPLE 2.7 Shape-regular meshes are easily shown to satisfy Assumption 2.6 with moderate $K, L, M$. Also, meshes which are anisotropically graded towards an edge typically lie in this class. A classical example of these arises in the approximation of boundary integral formulations of screen problems for elliptic PDEs, where the screen is a polygon. Near an edge, but away from the corners, the solution typically is badly behaved only in the direction orthogonal to the edge and efficient approximations require meshes which are anisotropically graded. 
For example, for the square screen $[0,1] \times[0,1]$, a typical tensor product anisotropic mesh would be: $\mathbf{x}_{i, j}=\left(t_{i}, t_{j}\right)$, where $t_{i}=(i / n)^{g} / 2$ and $t_{2 n-i}=1-(i / n)^{g} / 2$ for $i=0, \ldots, n$, for some grading exponent $g \geqslant 1$. (For example, see Noble, 1961; Petersdorff, 1989; Ervin et al., 1990; Graham et al., 1999.) An illustration of such a graded mesh is given in Fig. 2. In this case the elements become very long and thin near smooth parts of edges. In the $h p$ version of the finite-element method similar meshes but with more extreme grading may be used (e.g. Sauter \& Schwab, 1997) and these also satisfy Assumption 2.6.

We denote the class of meshes which satisfy Assumptions 2.2 and 2.6 as $\mathcal{M}_{D, E, K, L, M}$. From now on, if $A(\mathcal{T})$ and $B(\mathcal{T})$ are two mesh-dependent quantities, then the inequality $A(\mathcal{T}) \lesssim B(\mathcal{T})$ will mean that there is a constant $C$ independent of $\mathcal{T} \in \mathcal{M}_{D, E, K, L, M}$, such that $A(\mathcal{T}) \leqslant C B(\mathcal{T})$. (The class of meshes $\mathcal{M}_{D, E, K, L, M}$ depends on $D, E, K, L, M$, and we do not claim that $C$ is independent of $D, E, K, L, M$.) Also, the notation $A(\mathcal{T}) \sim B(\mathcal{T})$ will mean that $A(\mathcal{T}) \lesssim B(\mathcal{T})$ and $B(\mathcal{T}) \lesssim A(\mathcal{T})$.

Now we introduce finite-element spaces on the mesh $\mathcal{T}$.

Definition 2.8 (Finite-element spaces) For $m \geqslant 0$ and $\hat{\tau} \in\left\{\hat{\sigma}^{d}, \hat{\kappa}^{d}\right\}$, we define

$$
\mathbb{P}^{m}(\hat{\tau})= \begin{cases}\text { polynomials of total degree } \leqslant m \text { on } \hat{\tau} & \text { if } \hat{\tau}=\hat{\sigma}^{d}, \\ \text { polynomials of coordinate degree } \leqslant m \text { on } \hat{\tau} & \text { if } \hat{\tau}=\hat{\kappa}^{d} .\end{cases}
$$

Then we define

$$
\begin{aligned}
& \mathcal{S}_{0}^{m}(\mathcal{T})=\left\{u \in L^{\infty}(\Omega): u \circ \chi_{\tau} \in \mathbb{P}^{m}(\hat{\tau}), \tau \in \mathcal{T}\right\}, \text { for } m \geqslant 0 . \\
& \mathcal{S}_{1}^{m}(\mathcal{T})=\left\{u \in C^{0}(\Omega): u \circ \chi_{\tau} \in \mathbb{P}^{m}(\hat{\tau}), \tau \in \mathcal{T}\right\} \text { for } m \geqslant 1 .
\end{aligned}
$$

We finish this section with a generalization of a standard scaling argument which is used several times in later proofs.

Proposition 2.9 Let $\tau \in \mathcal{T}$ and let $\hat{t}$ be any simplex which is contained in the associated unit element $\hat{\tau} \in \mathbb{R}^{d}$. Let $\hat{P}$ denote any $d$-variate polynomial on $\hat{t}$ and define $t=\chi_{\tau}(\hat{t}), P=\hat{P} \circ \chi_{\tau}^{-1}$. Then for all $0 \leqslant s \leqslant 1$,

$$
\|P\|_{H^{s}(t)} \lesssim \rho_{\hat{t}}^{-s} \rho_{\tau}^{-s}\|P\|_{L^{2}(t)} .
$$

The hidden constant of proportionality in (2.4) depends on $\hat{P}$ only through its degree and is independent of $\hat{t}$.

Proof. The proof is a refinement of standard scaling arguments (e.g. Ciarlet, 1978).

Consider first the case $d=3$. Then $\Omega \subset \mathbb{R}^{3}$ is a bounded Euclidean domain and by the chain rule we have $\widehat{\nabla} \widehat{P}(\hat{\mathbf{x}})=J_{\tau}(\hat{\mathbf{x}})^{T}(\nabla P)\left(\chi_{\tau}(\hat{\mathbf{x}})\right)$, where $\widehat{\nabla}$ denotes the gradient with respect to $\hat{\mathbf{x}} \in \hat{\tau}$ and $\nabla$ denotes gradient with respect to $\mathbf{x} \in \tau$. By (2.1a), $J_{\tau}^{T} J_{\tau}$ is invertible and

$$
|P|_{H^{1}(t)}^{2}=\int_{\hat{t}}(\widehat{\nabla} \widehat{P})^{T}\left(J_{\tau}^{T} J_{\tau}\right)^{-1}(\widehat{\nabla} \widehat{P}) g_{\tau},
$$

where $|\cdot|_{H^{1}(t)}$ denotes the usual seminorm. Using (2.1b) we obtain

$$
|P|_{H^{1}(t)}^{2} \lesssim \rho_{\tau}^{-2} \int_{\hat{t}}|\widehat{\nabla} \widehat{P}|^{2} g_{\tau} .
$$


Since $\hat{t}$ is a simplex, we can also introduce an affine map $v: \hat{\sigma}^{3} \rightarrow \hat{t}$, introduce a new function $\widehat{P} \circ v$ and repeat the previous argument, using also Example 2.3 to obtain

$$
|P|_{H^{1}(t)}^{2} \lesssim \rho_{\tau}^{-2} \rho_{\hat{t}}^{-2} \int_{\hat{\sigma}^{3}}|\widetilde{\nabla}(\widehat{P} \circ v)|^{2} g g_{\tau},
$$

where $g$ is the Gram determinant for $v$ and $\widetilde{\nabla}$ denotes the gradient with respect to $\tilde{\mathbf{x}}:=v^{-1}(\hat{\mathbf{x}})$. Then, since $\hat{P} \circ v$ is a polynomial of the same degree as $\hat{P}$, by equivalence of norms on finite-dimensional spaces there follows

$$
|P|_{H^{1}(t)}^{2} \lesssim \rho_{\tau}^{-2} \rho_{\hat{t}}^{-2} \int_{\hat{\sigma}^{3}}|\widehat{P} \circ \nu|^{2} g g_{\tau}=\rho_{\tau}^{-2} \rho_{\hat{\tau}}^{-2}\|P\|_{L^{2}(t)}^{2} .
$$

(Here the hidden constant of proportionality depends on the degree of $\hat{P}$.) This proves the result for $s=1$. The intermediate values of $s$ are obtained by interpolation.

Turn now to the case $d=2$. When $\Omega$ is a bounded two-dimensional Euclidean domain, the proof is entirely analogous to that given above. Now consider the case when $\Omega$ is a piecewise smooth Lipschitz surface in $\mathbb{R}^{3}$. Since $\chi_{\tau}$ is assumed a smooth mapping, the element $\tau$ consists of a smooth portion of $\Omega$ and can be written $\tau=\eta(\tilde{\tau})$ where $\tilde{\tau} \subset \mathbb{R}^{2}$ is a planar, curvilinear element lying in one of the charts which determine $\Omega$ and $\eta$ is a smooth bijective map with smooth inverse. We consider $\eta$ as the transformation of the surface metric to a planar metric which is independent of the size of $\tau$. We may write the mapping $\chi_{\tau}$ as the composition $\chi_{\tau}=\eta \circ \chi_{\tilde{\tau}}$, where $\chi_{\tilde{\tau}}$ is now a scaling from the unit element $\hat{\tau}$ to $\tilde{\tau}$. Introduce the set $\tilde{t}:=\eta^{-1}(t) \subset \tilde{\tau}$ and the function $\tilde{P}:=P \circ \eta$ on $\tilde{\tau}$. The above result on two-dimensional Euclidean domains shows

$$
\|\tilde{P}\|_{H^{s}(\tilde{t})} \lesssim \rho_{\hat{t}}^{-s} \rho_{\tilde{\tau}}^{-s}\|\tilde{P}\|_{L^{2}(\tilde{t})} .
$$

Since the constants in $\rho_{\tilde{\tau}} \sim \rho_{\tau}$ only depend on the mapping $\eta$, and since we also have

$$
\|\tilde{P}\|_{H^{s}(\tilde{t})} \sim\|P\|_{H^{s}(t)}, \quad\|\tilde{P}\|_{L^{2}(\tilde{t})} \sim\|P\|_{L^{2}(t)},
$$

the result follows.

The argument in Proposition 2.9 can be extended to general positive $s$ when $\chi_{\tau}$ is simple enough, as the following extension shows.

COROLlary 2.10 Let the assumptions of Proposition 2.9 hold and suppose in addition that $\chi_{\tau}$ is an affine map. Then (2.4) holds uniformly for all $s$ in any compact subset of $(0, \infty)$.

Proof. Let $\alpha$ be any multi-index with $|\alpha|=k>1$. Then, repeating the argument by which we derived (2.7) from (2.5), we obtain

$$
\left\|\frac{\partial^{\alpha} P}{\partial x^{\alpha}}\right\|_{L_{2}(t)}^{2} \leqslant\left|\frac{\partial^{\beta} P}{\partial x^{\beta}}\right|_{H^{1}(t)}^{2} \lesssim \rho_{\tau}^{-2} \rho_{\hat{t}}^{-2} \int_{\hat{\sigma}^{3}}\left|\widetilde{\nabla}\left(\widehat{Q}_{\beta} \circ v\right)\right|^{2} g g_{\tau}
$$

where $\widehat{Q}_{\beta}(\hat{\mathbf{x}})=\left(\partial^{\beta} P / \partial x^{\beta}\right)\left(\chi_{\tau}(\hat{\mathbf{x}})\right), \hat{\mathbf{x}} \in \hat{\tau}$ and $\beta$ is a multi-index of order $|\beta|=k-1$.

Then, since both $\chi_{\tau}$ and $v$ are affine, $\widehat{Q}_{\beta} \circ v$ is still a polynomial of degree no more than the degree of $P$. Thus the equivalence of norms argument which was used to derive (2.8) can be applied again to obtain

$$
\left\|\frac{\partial^{\alpha} P}{\partial x^{\alpha}}\right\|_{L_{2}(t)}^{2} \lesssim \rho_{\tau}^{-2} \rho_{\hat{\tau}}^{-2} \sup _{|\beta|=k-1}\left\|\frac{\partial^{\beta} P}{\partial x^{\beta}}\right\|_{L^{2}(t)}^{2} .
$$


The required result (2.4) for any integer $s \geqslant 1$ follows by iterating this, with the intermediate values of $s$ being obtained by interpolation.

This generalizes the argument of Proposition 2.9 when $\tau$ is a subset of a Euclidean domain in $\mathbb{R}^{2}$ or $\mathbb{R}^{3}$. When $\tau$ is a surface element, the generalization of the proposition is trivial, since the assumption that $\chi_{\tau}$ is affine forces $\tau$ to be planar and the result follows from the $\mathbb{R}^{2}$ case.

\section{Inverse estimates}

In this section we prove our inverse estimates, which were motivated in the Introduction (see (1.3)). To define the scaling function $\rho$, recall the parameters $\rho_{\tau}$ introduced in Definition 2.1. From these we construct a continuous mesh function $\rho \in \mathcal{S}_{1}^{1}$ on $\Omega$ as follows.

Definition 3.1 (Mesh function) For each $p \in \mathcal{N}$, set $\rho_{p}=\max \left\{\rho_{\tau}: \mathbf{x}_{p} \in \bar{\tau}\right\}$. The mesh function $\rho$ is the unique function in $\mathcal{S}_{1}^{1}(\mathcal{T})$ such that $\rho\left(\mathbf{x}_{p}\right)=\rho_{p}$, for each $p \in \mathcal{N}$.

Clearly $\rho$ is a positive, continuous function on $\Omega$ and, by Assumption 2.6, it follows that $\rho(\mathbf{x}) \sim \rho_{\tau}$ for $\mathbf{x} \in \tau$, and all $\tau \in \mathcal{T}$. The main results of this section are Theorems 3.2, 3.4 and 3.6. The first two of these provide inverse estimates in positive Sobolev norms for functions $u \in \mathcal{S}_{i}^{m}(\mathcal{T})$ with continuity index $i=1,0$ respectively. The third theorem provides inverse estimates in negative norms.

Theorem 3.2 Let $0 \leqslant s \leqslant 1$ and $-\infty<\underline{\alpha}<\bar{\alpha}<\infty$. Then

$$
\left\|\rho^{\alpha} u\right\|_{H^{s}(\Omega)} \lesssim\left\|\rho^{\alpha-s} u\right\|_{L^{2}(\Omega)},
$$

uniformly in $\alpha \in[\underline{\alpha}, \bar{\alpha}], u \in \mathcal{S}_{1}^{m}(\mathcal{T})$.

REMARK 3.3 Since $\mathcal{S}_{1}^{m}(\mathcal{T}) \subset H^{s}(\Omega)$ for all $s<3 / 2$, it may be expected that the range of Sobolev indices for which Theorem 3.2 holds may be extended. Such an extension has been obtained in Dahmen et al. (2004) for shape-regular meshes at the expense of working in Besov norms. We have avoided such extensions here in order to simplify the present paper.

Proof. The proof is a generalization of Dahmen et al. (2004, Theorem 4.1). First we write

$$
\nabla\left(\rho^{\alpha} u\right)=\alpha \rho^{\alpha-1} u \nabla \rho+\rho^{\alpha} \nabla u .
$$

Using this, Assumption 2.6 and Proposition 2.9, we have

$$
\begin{aligned}
\left\|\nabla\left(\rho^{\alpha} u\right)\right\|_{L^{2}(\tau)}^{2} & \lesssim\left\|\rho^{\alpha-1} u\right\|_{L^{\infty}(\tau)}^{2}\|\nabla \rho\|_{L^{2}(\tau)}^{2}+\left\|\rho^{\alpha} \nabla u\right\|_{L^{2}(\tau)}^{2} \\
& \lesssim \rho_{\tau}^{2 \alpha-4}\|u\|_{L^{\infty}(\tau)}^{2}\|\rho\|_{L^{2}(\tau)}^{2}+\rho_{\tau}^{2 \alpha-2}\|u\|_{L^{2}(\tau)}^{2} \\
& \lesssim \rho_{\tau}^{2 \alpha-2}\|u\|_{L^{\infty}(\tau)}^{2}|\tau|+\rho_{\tau}^{2 \alpha-2}\|u\|_{L^{2}(\tau)}^{2} .
\end{aligned}
$$

Now a simple scaling argument shows that

$$
\|u\|_{L^{\infty}(\tau)}^{2}|\tau| \sim\|u\|_{L^{2}(\tau)}^{2} \quad \text { uniformly in } u \in S_{i}^{m}(\mathcal{T}), \quad i=0,1 .
$$

Hence

$$
\left\|\nabla\left(\rho^{\alpha} u\right)\right\|_{L^{2}(\tau)}^{2} \lesssim \rho_{\tau}^{2 \alpha-2}\|u\|_{L^{2}(\tau)}^{2} \sim\left\|\rho^{\alpha-1} u\right\|_{L^{2}(\tau)}^{2}
$$

and the proof for $s=1$ follows by summation over $\tau \in \mathcal{T}$. The proof for $s \in[0,1]$ follows by interpolation. 
Theorem 3.4 Let $0 \leqslant s<1 / 2$ and $-\infty<\underline{\alpha}<\bar{\alpha}<\infty$. Then

$$
\left\|\rho^{\alpha} u\right\|_{H^{s}(\Omega)} \lesssim\left\|\rho^{\alpha-s} u\right\|_{L^{2}(\Omega)},
$$

uniformly in $\alpha \in[\underline{\alpha}, \bar{\alpha}], u \in \mathcal{S}_{0}^{m}(\mathcal{T})$.

Proof. We give the proof for $d=2$. It is a generalization of Dahmen et al. (2004, Theorem 4.2). (The proof for $d=3$ follows similar lines.)

Firstly, it follows from a result of Faermann (2002, Lemma 3.1), that the fractional order Sobolev norm $\|\cdot\|_{H^{s}(\Omega)}$ admits an estimate in terms of local norms of the form

$$
\left\|\rho^{\alpha} u\right\|_{H^{s}(\Omega)}^{2} \lesssim \sum_{\tau \in \mathcal{T}}\left\{\rho_{\tau}^{2(\alpha-s)}\|u\|_{L^{2}(\tau)}^{2}+\sum_{\substack{\tau^{\prime} \in \mathcal{T} \\ \tau^{\prime} \cap \bar{\tau} \neq \emptyset}} \int_{\tau} \int_{\tau^{\prime}} \frac{\left|\left(\rho^{\alpha} u\right)(\mathbf{x})-\left(\rho^{\alpha} u\right)(\mathbf{y})\right|^{2}}{|\mathbf{x}-\mathbf{y}|^{2+2 s}} \mathrm{~d} x \mathrm{~d} y\right\} .
$$

(Note that in Faermann (2002) the estimate (3.2) is proved in a slightly different setting, where meshes on a surface $\Gamma$ are obtained by mappings of triangulations of the surface of a nearby polyhedron. However, it is easy to check that (3.2) still holds true in the more general setting of this paper.) Because of the local quasiuniformity (Assumption 2.6), the proof is finished, provided we can show

$$
\sum_{\tau \in \mathcal{T}} \sum_{\substack{\tau^{\prime} \in \mathcal{T} \\ \tau^{\prime} \cap \bar{\tau} \neq \emptyset}} \int_{\tau} \int_{\tau^{\prime}} \frac{\left|\left(\rho^{\alpha} u\right)(\mathbf{x})-\left(\rho^{\alpha} u\right)(\mathbf{y})\right|^{2}}{|\mathbf{x}-\mathbf{y}|^{2+2 s}} \mathrm{~d} x \mathrm{~d} y \lesssim \sum_{\tau \in \mathcal{T}} \rho_{\tau}^{2(\alpha-s)}\|u\|_{L^{2}(\tau)}^{2} .
$$

To prove this, we decompose the left-hand side of (3.3) as

$$
\sum_{\substack { \tau \in \mathcal{T} \\
\begin{subarray}{c}{\tau^{\prime} \in \mathcal{T} \backslash\{\tau\} \\
\tau^{\prime} \cap \bar{\tau} \neq \emptyset{ \tau \in \mathcal { T } \\
\begin{subarray} { c } { \tau ^ { \prime } \in \mathcal { T } \backslash \{ \tau \} \\
\tau ^ { \prime } \cap \overline { \tau } \neq \emptyset } }\end{subarray}} \int_{\tau} \int_{\tau^{\prime}} \frac{\left|\left(\rho^{\alpha} u\right)(\mathbf{x})-\left(\rho^{\alpha} u\right)(\mathbf{y})\right|^{2}}{|\mathbf{x}-\mathbf{y}|^{2+2 s}} \mathrm{~d} x \mathrm{~d} y+\sum_{\tau \in \mathcal{T}} \int_{\tau} \int_{\tau} \frac{\left|\left(\rho^{\alpha} u\right)(\mathbf{x})-\left(\rho^{\alpha} u\right)(\mathbf{y})\right|^{2}}{|\mathbf{x}-\mathbf{y}|^{2+2 s}} \mathrm{~d} x \mathrm{~d} y .
$$

By definition of the Aronszajn-Slobodeckij norm on $H^{s}(\tau)$ (see e.g. Faermann, 2002) and by using Proposition 2.9, the second term in (3.4) may be bounded by

$$
\sum_{\tau \in \mathcal{T}}\left\|\rho^{\alpha} u\right\|_{H^{s}(\tau)}^{2} \lesssim \sum_{\tau \in \mathcal{T}} \rho_{\tau}^{2(\alpha-s)}\|u\|_{L^{2}(\tau)}^{2} .
$$

Finally, following the proof of Dahmen et al. (2004, Theorem 4.2), the first term in (3.4) may be bounded by

$$
\sum_{\tau \in \mathcal{T}} \sum_{\substack{\tau^{\prime} \in \mathcal{T} \backslash\{\tau\} \\ \tau^{\prime} \cap \bar{\tau} \neq \emptyset}}\left\{\left\|\rho^{\alpha} u\right\|_{L^{\infty}(\tau)}^{2}+\left\|\rho^{\alpha} u\right\|_{L^{\infty}\left(\tau^{\prime}\right)}^{2}\right\} J_{\tau, \tau^{\prime}}, \quad \text { where } \mathcal{J}_{\tau, \tau^{\prime}}=\int_{\tau} \int_{\tau^{\prime}}|\mathbf{x}-\mathbf{y}|^{-2-2 s} \mathrm{~d} x \mathrm{~d} y .
$$

The proof may be completed by showing that, for all $\bar{\tau} \neq \overline{\tau^{\prime}}$ with $\bar{\tau} \cap \overline{\tau^{\prime}} \neq \emptyset$,

$$
\mathcal{J}_{\tau, \tau^{\prime}} \lesssim \min \left\{\rho_{\tau}^{-2 s}|\tau|, \rho_{\tau^{\prime}}^{-2 s}\left|\tau^{\prime}\right|\right\} .
$$


Inserting this into the left-hand side of (3.6) and using again Assumption 2.6 shows that the first term of (3.4) may be bounded by a constant times $\sum_{\tau \in \mathcal{T}}\|u\|_{L^{\infty}(\tau)}^{2}|\tau| \rho_{\tau}^{2 \alpha-2 s}$. Using (3.1), this can be bounded analogously to (3.5). Thus the proof is complete once we establish (3.7).

The estimate (3.7) follows from the proof of Theorem 4.2 in Dahmen et al. (2004) in the case when $\tau$ and $\tau^{\prime}$ are planar triangular elements. In this paper we allow the more general setting where $\tau$ and $\tau^{\prime}$ can be curved surface elements and the proof in (Dahmen et al., 2004) needs to be extended slightly.

First observe that since $\tau$ and $\tau^{\prime}$ are smooth subsurfaces of $\Omega$ (itself a piecewise smooth Lipschitz surface), $\tau \cup \tau^{\prime}$ may be parametrized by a bi-Lipschitz map $\eta: \overline{\tilde{\tau} \cup \tilde{\tau}^{\prime}}: \longrightarrow \overline{\tau \cup \tau^{\prime}}$, where $\tilde{\tau}=\eta^{-1}(\tau)$, $\tilde{\tau}^{\prime}=\eta^{-1}\left(\tau^{\prime}\right)$ and $\eta$ is independent of the mesh $\mathcal{T}$. Then

$$
\mathcal{J}_{\tau, \tau^{\prime}} \lesssim \int_{\tilde{\tau}} \int_{\tilde{\tau}^{\prime}}|\tilde{\mathbf{x}}-\tilde{\mathbf{y}}|^{-2-2 s} \mathrm{~d} \tilde{x} \mathrm{~d} \tilde{y} .
$$

Now, proceeding as in the proof of Dahmen et al. (2004, Theorem 4.2), it is easy to deduce from this that $\mathcal{J}_{\tau, \tau^{\prime}} \lesssim \int_{\tilde{\tau}} \operatorname{dist}(\tilde{\mathbf{y}}, \partial \tilde{\tau})^{-2 s} \mathrm{~d} \tilde{y}$. If we now define $\tilde{\chi}_{\tau}=\eta^{-1} \circ \chi_{\tau}$, and let $\tilde{J}_{\tau}$ denote the Jacobian of this map, it is easy to deduce (using (2.1a) and (2.1b)) that estimates (2.1a) and (2.1b) still hold when $J_{\tau}$ is replaced by $\tilde{\boldsymbol{J}}_{\tau}$. Hence, when $\tilde{\mathbf{y}}=\tilde{\chi}_{\tau}(\hat{\mathbf{y}})$, it follows that $\operatorname{dist}(\tilde{\mathbf{y}}, \partial \tilde{\tau}) \gtrsim \rho_{\tau} \operatorname{dist}(\hat{\mathbf{y}}, \partial \hat{\tau})$. Hence on using (2.1a) and (2.1b) for $\tilde{J}_{\tau}$, it follows that

$$
\mathcal{J}_{\tau, \tau^{\prime}} \lesssim\left\{\int_{\hat{\tau}} \operatorname{dist}(\hat{\mathbf{y}}, \partial \hat{\tau})^{-2 s} \mathrm{~d} \hat{y}\right\} \rho_{\tau}^{-2 s}|\tau| \lesssim \rho_{\tau}^{-2 s}|\tau|
$$

Since $\tau$ and $\tau^{\prime}$ are interchangeable, (3.7) follows.

The final theorem in this section (Theorem 3.6) provides estimates in negative Sobolev norms for finite-element functions. Before we prove this, we require the following technical lemma.

Lemma 3.5 Let $\hat{\tau}$ and $\mathbb{P}^{m}(\hat{\tau})$ be as in Definition 2.8. Then for each integer $m \geqslant 0$, there exists $\delta=$ $\delta(m) \in(0,1)$ with the following property:

For each $\hat{u} \in \mathbb{P}^{m}(\hat{\tau})$, there exists a simplex $\hat{t} \subset \hat{\tau}$ (which may depend on $u$ and $m$ ), such that

$$
\rho_{\hat{t}} \geqslant \delta \quad \text { and } \quad \inf _{\hat{\mathbf{x}} \in \hat{t}}|\hat{u}(\hat{\mathbf{x}})| \geqslant \delta\|\hat{u}\|_{L^{\infty}(\hat{\tau})}
$$

where $\rho_{\hat{t}}$ is as defined in Definition 2.1.

Proof. By equivalence of norms on finite-dimensional spaces, there exists $\gamma=\gamma(m)>0$ such that, for all $\hat{u} \in \mathbb{P}^{m}(\hat{\tau})$,

$$
\|\widehat{\nabla} \hat{u}\|_{L^{\infty}(\hat{\tau})} \leqslant \gamma\|\hat{u}\|_{L^{\infty}(\hat{\tau})}
$$

where $\widehat{\nabla}$ denotes the gradient with respect to $\hat{\mathbf{x}}$. Now, by choosing $\delta_{0}=\delta_{0}(m)$ such that $0<\delta_{0} \leqslant$ $(1+\gamma)^{-1}<1$, it follows that

$$
0<\delta_{0} \leqslant\left(1+\|\widehat{\nabla} \hat{u}\|_{L^{\infty}(\hat{\tau})} /\|\hat{u}\|_{L^{\infty}(\hat{\tau})}\right)^{-1}=\frac{\|\hat{u}\|_{L^{\infty}(\hat{\tau})}}{\|\widehat{\nabla} \hat{u}\|_{L^{\infty}(\hat{\tau})}+\|\hat{u}\|_{L^{\infty}(\hat{\tau})}}
$$

for all $\hat{u} \in \mathbb{P}^{m}(\hat{\tau})$. 
For any $\hat{\mathbf{x}} \in \mathbb{R}^{d}$ and $\rho>0$, let $B_{\rho}(\hat{\mathbf{x}})$ denote the open ball centred at $\hat{\mathbf{x}}$ with radius $\rho$. We shall establish the statement: For all $\hat{u} \in \mathbb{P}^{m}(\hat{\tau})$, there exists $\rho \geqslant \delta_{0}$ and $\hat{\mathbf{x}}^{*} \in \overline{\hat{\tau}}$ (both of which may depend on $u$ and $m$ ), such that

$$
\inf _{\hat{\mathbf{x}} \in B_{\rho}\left(\hat{\mathbf{x}}^{*}\right) \cap \overline{\hat{\tau}}}|\hat{u}(\hat{\mathbf{x}})| \geqslant \delta_{0}\|\hat{u}\|_{L^{\infty}(\hat{\tau})}
$$

Then, with a suitable choice of $\alpha \in(0,1)$, (depending only on the unit element $\hat{\tau}$ ), there is always a simplex $\hat{t} \subset B_{\rho}\left(\hat{\mathbf{x}}^{*}\right) \cap \overline{\hat{\tau}}$ with $\rho_{\hat{t}} \geqslant \alpha \delta_{0}$. The required result follows with $\delta=\alpha \delta_{0}$.

To establish (3.11), consider any $\hat{u} \in \mathbb{P}^{m}(\hat{\tau})$. Suppose that $\|\widehat{\nabla} \hat{u}\|_{L^{\infty}(\hat{\tau})} \neq 0$. Then $\|\hat{u}\|_{L^{\infty}(\hat{\tau})} \neq 0$ and we can choose $\rho=\rho(\hat{u}, m)>0$ by setting

$$
\rho=\left(1-\delta_{0}\right)\|\hat{u}\|_{L^{\infty}(\hat{\tau})} /\|\widehat{\nabla} \hat{u}\|_{L^{\infty}(\hat{\tau})} .
$$

By (3.10), we then have $\delta_{0} \leqslant \rho$. Moreover, if we now choose any $\hat{\mathbf{x}}^{*} \in \overline{\hat{\tau}}$ such that

$$
\left|\hat{u}\left(\hat{\mathbf{x}}^{*}\right)\right|=\|\hat{u}\|_{L^{\infty}(\hat{\tau})},
$$

then, for any $\hat{\mathbf{x}} \in B_{\rho}\left(\hat{\mathbf{x}}^{*}\right) \cap \overline{\hat{\tau}}$, we have

$$
\left|\hat{u}(\hat{\mathbf{x}})-\hat{u}\left(\hat{\mathbf{x}}^{*}\right)\right| \leqslant\|\widehat{\nabla} \hat{u}\|_{L^{\infty}(\hat{\tau})}\left|\hat{\mathbf{x}}-\hat{\mathbf{x}}^{*}\right|<\|\widehat{\nabla} \hat{u}\|_{L^{\infty}(\hat{\tau})} \rho=\left(1-\delta_{0}\right)\|\hat{u}\|_{L^{\infty}(\hat{\tau})}
$$

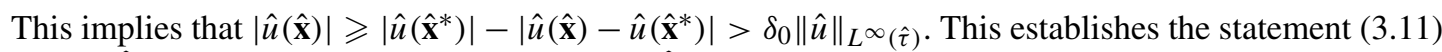
when $\|\widehat{\nabla} \hat{u}\|_{L^{\infty}(\hat{\tau})} \neq 0$. On the other hand, if $\|\widehat{\nabla} \hat{u}\|_{L^{\infty}(\hat{\tau})}=0$, then $\hat{u}$ is constant on $\hat{\tau}$, and (3.11) holds trivially with $\rho=\delta_{0}$ and any $\hat{\mathbf{x}}^{*} \in \overline{\hat{\tau}}$.

ThEOREM 3.6 Let $i \in\{0,1\}, m \geqslant i, 0 \leqslant s \leqslant 1$ and $-\infty<\underline{\alpha}<\bar{\alpha}<\infty$. Then the inequality

$$
\left\|\rho^{s+\alpha} u\right\|_{L^{2}(\Omega)} \lesssim\left\|\rho^{\alpha} u\right\|_{H^{-s}(\Omega)}
$$

holds uniformly in $u \in \mathcal{S}_{i}^{m}(\mathcal{T})$ and $\alpha \in[\underline{\alpha}, \bar{\alpha}]$. If $\chi_{\tau}$ is affine for all $\tau$ then (3.13) holds for all $0 \leqslant s \leqslant k$, where $k$ is as described in the second paragraph of Section 2.

Proof. The result is clear for $s=0$. Throughout the proof we make use of the inequality (2.4) which we assume holds for all $0 \leqslant s \leqslant k$. For $k>1$ the restriction that $\chi_{\tau}$ should be affine is required (see Corollary 2.10).

Suppose $u \in \mathcal{S}_{i}^{m}(\mathcal{T})$. The case $u \equiv 0$ is trivial, so from now on we assume that $u \not \equiv 0$. Then, for any $w \in H^{k}(\Omega)$, we have, by definition,

$$
\left\|\rho^{\alpha} u\right\|_{H^{-k}(\Omega)} \geqslant \frac{\left|\left(\rho^{\alpha} u, w\right)\right|}{\|w\|_{H^{k}(\Omega)}} .
$$

We shall construct $w \in H^{k}(\Omega)$ such that

$$
\left|\left(\rho^{\alpha} u, w\right)\right| \gtrsim\left\|\rho^{k+\alpha} u\right\|_{L^{2}(\Omega)}^{2}
$$

and

$$
\|w\|_{H^{k}(\Omega)} \lesssim\left\|\rho^{k+\alpha} u\right\|_{L^{2}(\Omega)},
$$


from which the result follows immediately.

The construction of $w$ is a generalization of the argument used to prove Theorem 4.7 in Dahmen et al. (2004). For any $\tau \in \mathcal{T}$, we have $u \circ \chi_{\tau} \in \mathbb{P}^{m}(\hat{\tau})$, and by Lemma 3.5, there exists a simplex $\hat{t}(\tau) \subseteq \hat{\tau}$ such that

$$
\rho_{\hat{t}(\tau)} \gtrsim 1 \quad \text { and } \quad \inf _{\hat{\mathbf{x}} \in \hat{t}(\tau)}\left|u \circ \chi_{\tau}(\hat{\mathbf{x}})\right| \geqslant \delta\left\|u \circ \chi_{\tau}\right\|_{L^{\infty}(\hat{\tau})} \gtrsim\left\|u \circ \chi_{\tau}\right\|_{L^{\infty}(\hat{\tau})} .
$$

(Recall that the constant $\delta$ in Lemma 3.5 was independent of $u$, hence $\delta \gtrsim 1$.) It is clear from this that $u \circ \chi_{\tau}$ does not change sign on $\hat{t}(\tau)$ and that

$$
|\hat{t}(\tau)| \sim 1 .
$$

Using the Bernstein representation of polynomials (as described, for example in Dahmen et al. (2004, Section 4.3)), we can construct a non-negative function $\hat{P}_{\hat{t}(\tau)}$ in $H_{0}^{k}(\hat{\tau})$ such that $\operatorname{supp} \hat{P}_{\hat{t}(\tau)}=\hat{t}(\tau), \hat{P}_{\hat{t}(\tau)}$ is a polynomial on $\hat{t}(\tau)$ and such that

$$
C_{2}|\hat{t}(\tau)|^{1 / p} \geqslant\left\|\hat{P}_{\hat{t}(\tau)}\right\|_{L^{p}(\hat{t}(\tau))} \geqslant C_{1}|\hat{t}(\tau)|^{1 / p},
$$

with $C_{1}, C_{2}$ independent of $p$ and of $\hat{t}(\tau)$. (This is done by constructing a positive-valued polynomial on $\hat{t}(\tau)$ which vanishes with sufficiently high order on the boundary of $\hat{t}(\tau)$.) Combining this with (3.17), we have

$$
\int_{\hat{t}(\tau)} \hat{P}_{\hat{t}(\tau)} \sim|\hat{t}(\tau)| \sim 1
$$

Now set $t(\tau)=\chi_{\tau}(\hat{t}(\tau)) \subseteq \tau$ and define a corresponding non-negative function $P_{t(\tau)} \in H^{k}(\Omega)$ by setting $P_{t(\tau)}=\hat{P}_{\hat{t}(\tau)} \circ \chi_{\tau}^{-1}$ on $\tau$ and $P_{t(\tau)}=0$ on $\Omega \backslash \tau$. It follows that

$$
\operatorname{supp} P_{t(\tau)}=t(\tau) \quad \text { and } \quad \int_{t(\tau)} P_{t(\tau)} \sim|\tau| .
$$

(The proof of the second relation makes use of (2.1a) and (3.19).)

For each $\tau \in \mathcal{T}$, we introduce scalars

$$
b_{\tau}=\rho_{\tau}^{k+\alpha} \operatorname{sign}\left(\left.u\right|_{t(\tau)}\right) \inf _{\mathbf{x} \in t(\tau)}|u(\mathbf{x})|,
$$

and we define $w \in H^{k}(\Omega)$ by

$$
w=\sum_{\tau \in \mathcal{T}} b_{\tau} \rho_{\tau}^{k} P_{t(\tau)}
$$

Then, using (3.22), (3.20), we obtain

$$
\left(\rho^{\alpha} u, w\right)=\sum_{\tau \in \mathcal{T}} \int_{t(\tau)}\left(\rho_{\tau} / \rho\right)^{k}\left\{\rho^{k+\alpha} b_{\tau} u\right\} P_{t(\tau)} .
$$

By (3.21), (2.3a) and the non-negativity of $P_{t(\tau)}$, we have

$$
\left|\left(\rho^{\alpha} u, w\right)\right| \gtrsim \sum_{\tau \in \mathcal{T}} \rho_{\tau}^{2(k+\alpha)}\left\{\inf _{\mathbf{x} \in t(\tau)}|u(\mathbf{x})|\right\}^{2} \int_{t(\tau)} P_{t(\tau)} .
$$


Then, by (3.16) and (3.20),

$$
\left|\left(\rho^{\alpha} u, w\right)\right| \gtrsim \sum_{\tau \in \mathcal{T}} \rho_{\tau}^{2(k+\alpha)}\|u\|_{L^{\infty}(\tau)}^{2}|\tau|,
$$

which, using (3.1) and (2.3a), readily yields (3.14).

To obtain (3.15), we first obtain the estimate

$$
\|w\|_{H^{k}(\Omega)}^{2}=\sum_{\tau \in \mathcal{T}}\|w\|_{H^{k}(\tau)}^{2} \leqslant \sum_{\tau \in \mathcal{T}} \rho_{\tau}^{2 k}\left|b_{\tau}\right|^{2}\left\|P_{t(\tau)}\right\|_{H^{k}(t(\tau))}^{2} \lesssim \sum_{\tau \in \mathcal{T}}\left|b_{\tau}\right|^{2}\left\|P_{t(\tau)}\right\|_{L^{2}(t(\tau))}^{2},
$$

where the final inequality follows from Proposition 2.9 (or Corollary 2.10) and (3.16). Since

$$
\left\|P_{t(\tau)}\right\|_{L^{2}(t(\tau))}^{2}=\int_{\hat{t}(\tau)}\left|\hat{P}_{\hat{t}(\tau)}\right|^{2} g_{\tau} \sim|\tau| \int_{\hat{t}(\tau)}\left|\hat{P}_{\hat{t}(\tau)}\right|^{2}
$$

(3.18), and then (3.17), yields $\left\|P_{t(\tau)}\right\|_{L^{2}(t(\tau))}^{2} \sim|\tau||\hat{t}(\tau)| \sim|t(\tau)|$. Using this together with the definition (3.21) of $b_{\tau}$, we finally obtain

$$
\|w\|_{H^{k}(\Omega)}^{2} \lesssim \sum_{\tau \in \mathcal{T}}\left|b_{\tau}\right|^{2}|t(\tau)| \leqslant \sum_{\tau \in \mathcal{T}} \rho_{\tau}^{2(k+\alpha)}\left\{\inf _{\mathbf{x} \in t(\tau)}|u(\mathbf{x})|\right\}^{2}|t(\tau)| \lesssim\left\|\rho^{k+\alpha} u\right\|_{L^{2}(\Omega)}^{2},
$$

i.e. (3.15).

REMARK 3.7 When $i=k=1$, a simpler construction for $w$ can be given in terms of a suitable element in $\mathcal{S}_{1}^{m}(\mathcal{T})$ (see Graham et al., 2000a for the case $m=1$ ).

REMARK 3.8 Note that the test function $w$ constructed in the proof of Theorem 3.6 vanishes at the boundaries of elements. Hence if $\Omega$ has a boundary then $w$ belongs to the closure of the space $C_{0}^{\infty}(\Omega)$ with respect to the $H^{s}(\Omega)$ norm (this space is usually denoted $H_{0}^{s}(\Omega)$ ). Thus the result of Theorem 6 also holds if $H^{-s}(\Omega)$ was defined as the dual of $H_{0}^{s}(\Omega)$, although we have not so defined it here.

\section{Galerkin boundary element method}

In this section we review briefly the Galerkin boundary element method for elliptic PDEs, which forms the basis of the applications in the proceeding sections. We consider a $2 \mathrm{D}$ surface $\Omega$ in $\mathbb{R}^{3}$ (i.e. the case $d=2$ above). To conform with more usual notation in boundary integral equations, we rename this surface $\Gamma$. To avoid technicalities, we assume that $\Gamma$ is a closed bounded Lipschitz surface in $\mathbb{R}^{3}$, consisting of infinitely smooth (i.e. $\mathcal{C}^{\infty}$ ) pieces joined at corners and edges. (The extension to the case when $\Gamma$ is an open surface may require slightly different Sobolev spaces. Although this extension is possible, in the interests of brevity, we do not give it here.)

Consider the general linear integral equation

$$
(\lambda I+\mathcal{K}) u(\mathbf{x}):=\lambda u(\mathbf{x})+\int_{\Gamma} k(\mathbf{x}, \mathbf{y}) u(\mathbf{y}) \mathrm{d} y=g(\mathbf{x}), \quad \mathbf{x} \in \Gamma,
$$

for some given scalar $\lambda \in \mathbb{R}$, kernel function $k$ and sufficiently smooth right-hand side $g$. The corresponding weak form is

$$
\text { Find } u \in \mathcal{V}^{\mu} \text { such that } a(u, v):=((\lambda I+\mathcal{K}) u, v)=(g, v) \text { for all } v \in \mathcal{V}^{\mu} \text {. }
$$


In general the energy space $\mathcal{V}^{\mu}$ is a closed subspace of $H^{\mu}(\Gamma)$ for some $\mu \in \mathbb{R}$ with norm induced by $H^{\mu}(\Gamma)$. (The most usual case is $\mathcal{V}^{\mu}=H^{\mu}(\Gamma)$.) The bracket $(\cdot, \cdot)$ denotes the continuous extension of the $L^{2}(\Gamma)$ scalar product to the $H^{-\mu}(\Gamma) \times H^{\mu}(\Gamma)$ duality pairing. Typical examples of kernels $k$ and scalars $\lambda$ in (4.1) arise in boundary integral reformulations of the PDE problem

$$
-\Delta u+\omega^{2} u=0,
$$

for some (generally complex) parameter $\omega$, subject to Dirichlet or Neumann boundary conditions on $\Gamma$ (together with appropriate decay conditions at infinity if the computational domain is unbounded). The fundamental solution for (4.3) is $G(\mathbf{z}):=\mathrm{e}^{-\omega|\mathbf{z}|} /(4 \pi|\mathbf{z}|)$ and equations (4.1) appear in three standard formats:

$$
\begin{aligned}
\text { Single layer potential: } & k(\mathbf{x}, \mathbf{y})=G(\mathbf{x}-\mathbf{y}), \quad \lambda=0 \\
\text { Double layer potential: } & k(\mathbf{x}, \mathbf{y})=\partial / \partial n(\mathbf{y}) G(\mathbf{x}-\mathbf{y}), \lambda= \pm 1 / 2 \\
\text { Hypersingular operator: } & k(\mathbf{x}, \mathbf{y})=\partial / \partial n(\mathbf{x}) \partial / \partial n(\mathbf{y}) G(\mathbf{x}-\mathbf{y}), \lambda=0 .
\end{aligned}
$$

It is well known that existence, uniqueness and well posedness of the weak problem (4.2) follows if the following three conditions hold:

Continuity: There exists $C>0$ such that

$$
\forall u, v \in \mathcal{V}^{\mu}:|a(u, v)| \leqslant C\|u\|_{H^{\mu}(\Gamma)}\|v\|_{H^{\mu}(\Gamma)} .
$$

Gårding's inequality: There exist a constant $\gamma>0$ and a compact operator $T: \mathcal{V}^{\mu} \rightarrow\left(\mathcal{V}^{\mu}\right)^{\prime}$ with associated bilinear form $t(\cdot, \cdot)=(T \cdot, \cdot)$ such that

$$
\forall u \in \mathcal{V}^{\mu}: a(u, u) \geqslant \gamma\|u\|_{H^{\mu}(\Gamma)}^{2}-t(u, u)
$$

Injectivity:

$$
\text { If } u \in \mathcal{V}^{\mu} \text {, then } a(u, v)=0 \forall v \in \mathcal{V}^{\mu} \quad \text { implies } u=0 .
$$

Let us recall what is known about the conditions (4.5) for the operators (4.4), in the special case when $\omega \in[0, \infty)$ in (4.3). (Proofs are given, e.g., in Sauter \& Schwab, 2004.) The single-layer potential (4.4a), satisfies (4.5) in space $\mathcal{V}^{\mu}=H^{-1 / 2}(\Gamma)$. The double-layer potential (4.4b) is known to satisfy (4.5) in $\mathcal{V}^{\mu}=L^{2}(\Gamma)$ provided $\Gamma$ is a sufficiently smooth (e.g. Lyapunov) surface. In this case the generalization to less smooth surfaces (e.g. polyhedra) is still an open problem - see Elschner (1992). Subject to the further restriction that $\omega>0$, the hypersingular operator (4.4c) satisfies (4.5) in $\mathcal{V}^{\mu}=H^{1 / 2}(\Gamma)$.

REMARK 4.1 The case of the Laplace operator $(\omega=0)$ is exceptional for the hypersingular operator, since, in order to ensure (4.5), the energy space $\mathcal{V}^{\mu}$ has to be chosen as the quotient space $H^{1 / 2}(\Gamma) / \mathbb{R}$. In order to reduce technicalities we will always assume in the following that

$$
\text { conditions (4.5) hold in } \mathcal{V}^{\mu}=H^{\mu}(\Gamma) \text { for some } \mu \in\{-1 / 2,0,1 / 2\} \text {. }
$$

Under this restriction, our analysis only handles the hypersingular operator for the case $\omega>0$. Our analysis could be further extended to the case $\omega=0$ (or indeed to integral equation formulations of the Helmholtz equation ((4.3) with $\omega$ purely imaginary), but for brevity we do not do that here. 
In the (conforming) Galerkin method we select a space of piecewise polynomial functions $\mathcal{S}_{i}^{m}(\mathcal{T}) \subset$ $H^{\mu}(\Gamma)$ and approximate (4.2) by seeking $U \in \mathcal{S}_{i}^{m}(\mathcal{T})$, such that

$$
a(U, V)=(g, V) \quad \text { for all } V \in \mathcal{S}_{i}^{m}(\mathcal{T}) .
$$

Note that when $\mu<1 / 2$ we may choose $i=0$ or 1 , but when $\mu \geqslant 1 / 2$ we are forced to work in the space of continuous piecewise polynomials $(i=1)$.

In order to realize (4.6) numerically, we need to introduce a basis for $\mathcal{S}_{i}^{m}(\mathcal{T})$. For convenience we shall restrict here to standard nodal bases defined as follows. For each $\tau \in \mathcal{T}$, we define (see Definition 2.8)

$$
\mathbb{P}^{m}(\tau)=\left\{u: \tau \rightarrow \mathbb{R}:\left.u \circ \chi_{\tau}\right|_{\hat{\tau}} \in \mathbb{P}^{m}(\hat{\tau})\right\}
$$

In $\overline{\hat{\tau}}$, we choose a set of $d(m):=(m+1)(m+2) / 2$ nodes $\left\{\hat{\mathbf{x}}_{p}: p=1, \ldots, d(m)\right\}$ so that each $\hat{u} \in \hat{\mathbb{P}}^{m}(\hat{\tau})$ is uniquely determined by its values at the $\hat{\mathbf{x}}_{p}$. (This is the so-called unisolvence property. Some of the $\hat{\mathbf{x}}_{p}$ may lie on the boundary of $\overline{\hat{\tau}}$.) The corresponding nodes on $\bar{\tau}$ are defined by: $\mathbf{x}_{p, \tau}:=\chi_{\tau}\left(\hat{\mathbf{x}}_{p}\right)$ and there exist corresponding nodal basis functions $\phi_{p, \tau} \in \mathbb{P}^{m}(\tau) \in \mathcal{S}_{0}^{m}(\mathcal{T})$, with the property that

$$
\phi_{p, \tau}\left(\mathbf{x}_{q, \tau^{\prime}}\right)=\delta_{(p, \tau),\left(q, \tau^{\prime}\right)}, \text { for } p, q=1, \ldots, d(m), \tau, \tau^{\prime} \in \mathcal{T} .
$$

The functions

$$
\left\{\phi_{p, \tau}: p=1, \ldots d(m), \tau \in \mathcal{T}\right\}
$$

then constitute a suitable basis of $\mathcal{S}_{0}^{m}(\mathcal{T})$.

For $\mathcal{S}_{1}^{m}(\mathcal{T})$, we require further that if two elements $\tau$ and $\tau^{\prime}$ share a common edge $e$, then, this edge is parametrized equally from both sides. More precisely, we require that if $\chi_{\tau}^{-1}(e)=\hat{e}$ and $\chi_{\tau^{\prime}}^{-1}(e)=\hat{e}^{\prime}$ then there exists an affine mapping $\gamma: \hat{e} \rightarrow \hat{e}^{\prime}$ such that $\chi_{\tau}$ and $\chi_{\tau^{\prime}} \circ \gamma$ coincide pointwise on $\hat{e}$. We assume that the points $\mathbf{x}_{p, \tau}$ and $\mathbf{x}_{p, \tau^{\prime}}$ restricted to $e$ coincide and that the values of $u$ at these points are sufficient to determine uniquely $\left.u\right|_{e}$ on $e$. In this case any $u \in \mathcal{S}_{1}^{m}(\mathcal{T})$ is determined uniquely by its values at the set of global nodes, namely the set $\left\{\mathbf{x}_{p, \tau}: p=1, \ldots, d(m), \tau \in \mathcal{T}\right\}$, where nodes on the boundaries of several elements now constitute a single 'freedom'. Denoting this set more abstractly by $\left\{\mathbf{x}_{f}: f \in \mathcal{F}\right\}$ for some suitable index set of freedoms $\mathcal{F}$, then our basis for $\mathcal{S}_{1}^{m}(\mathcal{T})$ is

$$
\left\{\phi_{f}: f \in \mathcal{F}\right\},
$$

where $\phi_{f} \in \mathcal{S}_{1}^{m}(\mathcal{T})$ is the unique function satisfying

$$
\phi_{f}\left(\mathbf{x}_{f^{\prime}}\right)=\delta_{f, f^{\prime}}, \text { for all } f, f^{\prime} \in \mathcal{F} .
$$

Clearly the basis (4.8) may be written in the abstract form (4.9) by allowing the set $\mathcal{F}$ to contain double indices of the form $(p, \tau)$. With this notation, (4.10) follows from (4.7). Moreover, in any case,

$$
\operatorname{supp} \phi_{f} \subseteq \bigcup\left\{\bar{\tau}: \mathbf{x}_{f} \in \bar{\tau}\right\}
$$

Note also that, because of the above unisolvence assumption above,

$$
\text { the functional } \hat{u} \longmapsto\left\{\sum_{p=1}^{d(m)}\left|\hat{u}\left(\hat{\mathbf{x}}_{p}\right)\right|^{2}\right\}^{1 / 2} \quad \text { is a norm on } \mathbb{P}^{m}(\hat{\tau}) .
$$


Writing $U=\sum_{f^{\prime} \in \mathcal{F}} U_{f^{\prime}} \phi_{f^{\prime}}$, (4.6) is equivalent to the linear system

$$
\sum_{f^{\prime} \in \mathcal{F}}\left(\lambda M_{f, f^{\prime}}+K_{f, f^{\prime}}\right) U_{f^{\prime}}=g_{f}, \quad f \in \mathcal{F},
$$

where $g_{f}=\left(g, \phi_{f}\right), M_{f, f^{\prime}}=\left(\phi_{f^{\prime}}, \phi_{f}\right)$ is the mass matrix and

$$
K_{f, f^{\prime}}=\int_{\Gamma} \int_{\Gamma} k(\mathbf{x}, \mathbf{y}) \phi_{f^{\prime}}(\mathbf{y}) \phi_{f}(\mathbf{x}) \mathrm{d} y \mathrm{~d} x, \quad f, f^{\prime} \in \mathcal{F} .
$$

is the stiffness matrix. The mass matrix $M$ is sparse and can be easily computed. The stiffness matrix is dense and generally has to be approximated by quadrature. Replacing $K_{f, f^{\prime}}$ by an approximation $\tilde{K}_{f, f^{\prime}}$ leads to the discrete counterpart of (4.6): Find $\tilde{U} \in \mathcal{S}_{i}^{m}(\mathcal{T})$, such that

$$
\tilde{a}(\tilde{U}, V)=(f, V), \quad \text { for all } V \in \mathcal{S}_{i}^{m}(\mathcal{T}),
$$

where

$$
\tilde{a}(V, W):=\sum_{f \in \mathcal{F}} \sum_{f^{\prime} \in \mathcal{F}} W_{f}\left(\lambda M_{f, f^{\prime}}+\tilde{K}_{f, f^{\prime}}\right) V_{f^{\prime}}
$$

The stability and convergence of $\tilde{U}$ is provided by the first 'Strang lemma'. A version for symmetric continuous elliptic bilinear forms is given in Ciarlet (1978); however in this paper we need an extension to forms satisfying the weaker assumptions (4.5). Before stating the result, we first introduce the relevant parameters. A measure for stability of the perturbed bilinear form is

$$
r_{\text {stab }}:=\sup _{V, W \in \mathcal{S}_{i}^{m}(\mathcal{T}) \backslash\{0\}} \frac{|a(V, W)-\tilde{a}(V, W)|}{\|V\|_{H^{\mu}(\Gamma)}\|W\|_{H^{\mu}(\Gamma)}} .
$$

The stability of the perturbed problem (4.15) follows if $r_{\text {stab }}$ approaches 0 as the mesh is refined. However, the rate of convergence of $\tilde{U}$ may depend on the regularity parameter $\delta$ in the following assumption:

$$
\text { the solution } u \text { of (4.2) satisfies } u \in H^{\mu+\delta}(\Gamma), \quad \text { for some } \delta \geqslant 0 \text {. }
$$

Note that conditions (4.5) ensure that (4.18) holds at least for $\delta=0$.

With the regularity assumption in place, the rate of convergence depends on the quantity

$$
r_{\text {conv }}:=\sup _{V, W \in \mathcal{S}_{i}^{m}(\mathcal{T}) \backslash\{0\}} \frac{|a(V, W)-\tilde{a}(V, W)|}{\|V\|_{H^{v}(\Gamma)}\|W\|_{H^{\mu}(\Gamma)}},
$$

where the parameter $v$ has to satisfy

$$
\mu \leqslant v \leqslant \mu+\delta \quad \text { with } \quad \mathcal{S}_{i}^{m}(\mathcal{T}) \subset H^{v}(\Gamma) .
$$

Lemma 4.2 Consider the problem (4.2), where $a(\cdot, \cdot)$ satisfies (4.5). Suppose

$$
r_{\text {stab }} \rightarrow 0 \quad \text { as } \quad h:=\max \left\{h_{\tau}: \tau \in \mathcal{T}\right\} \rightarrow 0 .
$$


Then, for sufficiently small $h$, the approximate Galerkin method (4.15) has a unique solution $\tilde{U} \in$ $\mathcal{S}_{i}^{m}(\mathcal{T})$. If, in addition, (4.18) holds and $v$ satisfies (4.20), then we have the error estimate

$$
\|u-\tilde{U}\|_{H^{\mu}(\Gamma)} \lesssim\left(\sup _{\substack{w \in H^{2 v-\mu}(\Gamma) \\\|w\|_{H^{2 v-\mu}(\Gamma)}=1}} \inf _{Z \in \mathcal{S}_{i}^{m}(\mathcal{T})}\|w-Z\|_{H^{v}(\Gamma)}\right) \inf _{Z \in \mathcal{S}_{i}^{m}(\mathcal{T})}\|u-Z\|_{H^{v}(\Gamma)}+r_{\text {conv }}\|u\|_{H^{v}(\Gamma)} .
$$

The proof of this lemma can be found in Sauter \& Schwab (2004, Satz 4.2.11 and Bemerkung 4.2.12).

REMARK 4.3 Note that since $\mu \leqslant v$, we always have $r_{\text {conv }} \leqslant r_{\text {stab }}$, and the best possible estimate for $r_{\text {conv }}$ would be obtained by choosing the maximal $v$ so that $\mathcal{S}_{i}^{m}(\mathcal{T}) \subset H^{v}(\Gamma)$, provided also that $\mu \leqslant v \leqslant \mu+\delta$. The error estimate (4.22) will then give a rate of convergence which is the maximum of $r_{\text {conv }}$ and the first term in the right-hand side of (4.22).

In the applications in the following two sections we shall study two different stiffness matrix approximations $\tilde{K}$ to $K$. Our estimates for the induced perturbation in the bilinear form do not depend on the underlying energy space and in their simplest possible form imply

$$
|a(V, W)-\tilde{a}(V, W)| \leqslant C_{h}\|V\|_{L^{2}(\Gamma)}\|W\|_{L^{2}(\Gamma)}, \quad \text { for all } V, W \in \mathcal{S}_{i}^{m}(\mathcal{T}),
$$

where $C_{h} \rightarrow 0$ as $h:=\max \left\{h_{\tau}: \tau \in \mathcal{T}\right\} \rightarrow 0$.

Lebesgue-type norms (here $L_{2}$, but they may be $L_{1}$ ) naturally appear in the right-hand side (see (5.2) and (6.11)). In order to use (4.23) to estimate $r_{\text {stab }}$ and $r_{\text {conv }}$, the argument differs according to whether $\mu \geqslant 0$ or $\mu<0$.

If $\mu \geqslant 0$, then the estimates

$$
r_{\text {stab }} \leqslant C_{h} \quad \text { and } \quad r_{\text {conv }} \leqslant C_{h} \quad \text { for all } \mu \leqslant v \leqslant \mu+\delta
$$

are a trivial consequence and cannot be bettered. Thus the rate of convergence provable from (4.22) is limited to $C_{h}$ in this case. Thus, for the double-layer potential and the hypersingular operator the simplest choice $v=\mu$ and $\delta=0$ is optimal in Lemma 4.2, leading to $r_{\text {conv }}=r_{\text {stab }}$, independent of any regularity in the problem.

The situation is different if $\mu<0$, since then inverse estimates are needed to estimate the $L_{2}$ norms in the right-hand side of (4.23) in terms of (negative) energy norms. Since inverse estimates incur some blow-up as $h \rightarrow \infty$ there is the possibility of obtaining a better estimate for $r_{\text {conv }}$ than for $r_{\text {stab }}$, when some regularity is present. This is the gain from the use of different measures for stability and consistency.

For the single-layer operator, $\mu=-1 / 2$ and one has to combine inverse inequalities for $V$ and

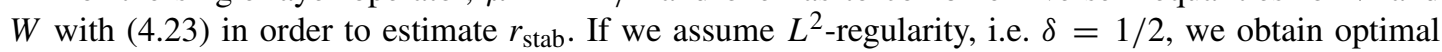
estimates for $r_{\text {conv }}$ by choosing $v=0$ in (4.20). Under moderate assumptions on the mesh we have

$$
\sup _{\substack{w \in H^{1 / 2}(\Gamma) \\\|w\|_{H^{1 / 2}(\Gamma)}=1}} \inf _{Z \in \mathcal{S}_{i}^{m}(\mathcal{T})}\|w-Z\|_{L^{2}(\Gamma)} \leqslant C h^{1 / 2}
$$


and the error estimate (4.22) takes the form

$$
\|u-\tilde{U}\|_{H^{-1 / 2}(\Gamma)} \lesssim h^{1 / 2} \inf _{Z \in \mathcal{S}_{i}^{m}(\mathcal{T})}\|u-Z\|_{L^{2}(\Gamma)}+r_{\text {conv }}\|u\|_{L^{2}(\Gamma)} .
$$

Since the estimate of $r_{\text {conv }}$ only requires one application of inverse estimates (for the function $W$ ), the term $r_{\text {conv }}$ should converge faster to zero than $r_{\text {stab }}$ and estimate (4.24) should be better than the 'trivial' one (corresponding to the choice $v=-1 / 2$ ):

$$
\|u-\tilde{U}\|_{H^{-1 / 2}(\Gamma)} \lesssim \inf _{Z \in \mathcal{S}_{i}^{m}(\mathcal{T})}\|u-Z\|_{H^{-1 / 2}(\Gamma)}+r_{\text {stab }}\|u\|_{H^{-1 / 2}(\Gamma)} .
$$

This is the reason why sharp inverse estimates play a key role in the analysis. We give detailed estimates for $r_{\text {stab }}$ and $r_{\text {cond }}$ in the following sections.

\section{Galerkin method with quadrature}

The effect of quadrature errors in Galerkin methods is analysed in Graham et al. (2000a), under the assumption of shape-regular meshes. The following theory generalizes these results, allowing also the treatment of degenerate mesh sequences, provided they satisfy Assumptions 2.2 and 2.6.

THEOREM 5.1 Suppose, for all $f, f^{\prime} \in \mathcal{F}$ the approximate matrix entries $\tilde{K}_{f, f^{\prime}}$ are constructed so that the following error estimate holds:

$$
\left|K_{f, f^{\prime}}-\tilde{K}_{f, f^{\prime}}\right| \lesssim h^{\chi+1}\left|\operatorname{supp} \phi_{f}\right|\left|\operatorname{supp} \phi_{f^{\prime}}\right|
$$

for some $\chi \geqslant 0$ where $h=\max \left\{h_{\tau}: \tau \in \mathcal{T}\right\}$ is the global mesh diameter. Let $i \in\{0,1\}$. Then, for all $v_{1}, v_{2} \in[-1, k]$ such that $\mathcal{S}_{i}^{m}(\mathcal{T}) \subset H^{\max \left\{v_{1}, v_{2}\right\}}(\Gamma)$,

$$
\frac{|a(V, W)-\tilde{a}(V, W)|}{\|V\|_{H^{\nu_{1}}(\Gamma)}\|W\|_{H^{v_{2}}(\Gamma)}} \lesssim h^{\chi+1} \sqrt{\sum_{\tau \in \mathcal{T}} \rho_{\tau}^{2 v_{1}^{-}}|\tau|} \sqrt{\sum_{\tau \in \mathcal{T}} \rho_{\tau}^{2 v_{2}^{-}}|\tau|}
$$

uniformly in $V, W \in \mathcal{S}_{i}^{m}(\mathcal{T})$ where $v_{i}^{-}:=\min \left\{v_{i}, 0\right\}$.

REMARK 5.2 Theorem 5.1 extends to all $v_{1}, v_{2} \in[-k, k]$ under the assumption that $\chi_{\tau}$ is affine for each $\tau \in \mathcal{T}$ (see Theorem 3.6).

Proof. By the definitions (4.2) and (4.16) of $a$ and $\tilde{a}$, we have, for all $V, W \in \mathcal{S}_{i}^{m}(\mathcal{T})$,

$|a(V, W)-\tilde{a}(V, W)| \leqslant \sum_{f \in \mathcal{F}} \sum_{f^{\prime} \in \mathcal{F}}\left|V_{f}\right|\left|K_{f, f^{\prime}}-\tilde{K}_{f, f^{\prime}}\right|\left|W_{f^{\prime}}\right| \leqslant h^{\chi+1}\left\{\sum_{f \in \mathcal{F}} s_{f}\left|V_{f}\right|\right\}\left\{\sum_{f \in \mathcal{F}} s_{f}\left|W_{f}\right|\right\}$,

where $s_{f}=\left|\operatorname{supp} \phi_{f}\right|$. Now, using the Cauchy-Schwarz inequality, we obtain

$$
\sum_{f \in \mathcal{F}} s_{f}\left|V_{f}\right|=\sum_{f \in \mathcal{F}}\left\{\rho_{f}^{v_{1}^{-}} s_{f}^{1 / 2}\right\}\left\{\rho_{f}^{-v_{1}^{-}} s_{f}^{1 / 2}\left|V_{f}\right|\right\} \leqslant \sqrt{\sum_{f \in \mathcal{F}} \rho_{f}^{2 v_{1}^{-}} s_{f}} \sqrt{\sum_{f \in \mathcal{F}} \rho_{f}^{-2 v_{1}^{-}} s_{f}\left|V_{f}\right|^{2}}
$$


where $\rho_{f}=\rho\left(\mathbf{x}_{f}\right)$ and $\rho$ is the piecewise linear function specified in Definition 3.1.

Now, using (4.11) and then (2.3a) and (2.3b), we have

$$
\sum_{f \in \mathcal{F}} \rho_{f}^{2 v_{1}^{-}} s_{f} \leqslant \sum_{f \in \mathcal{F}} \rho_{f}^{2 v_{1}^{-}} \sum_{\substack{\tau \in \mathcal{T} \\ \mathbf{x} f \in \bar{\tau}}}|\tau|=\sum_{\tau \in \mathcal{T}} \sum_{\substack{f \in \mathcal{F} \\ \mathbf{x}_{f} \in \bar{\tau}}} \rho_{f}^{2 v_{1}^{-}}|\tau| \lesssim \sum_{\tau \in \mathcal{T}} \rho_{\tau}^{2 v_{1}^{-}}|\tau|,
$$

where the constant of proportionality in the last inequality depends on the polynomial degree $m$, but not on the mesh $\mathcal{T}$ or on $v_{1}$. A similar argument shows that

$$
\sum_{f \in \mathcal{F}} \rho_{f}^{-2 v_{1}^{-}} s_{f}\left|V_{f}\right|^{2} \lesssim \sum_{\tau \in \mathcal{T}} \rho_{\tau}^{-2 v_{1}^{-}} \sum_{\substack{f \in \mathcal{F} \\ x_{f} \in \bar{\tau}}}\left|V_{f}\right|^{2}|\tau|
$$

Thus, by a simple scaling argument based on (4.12) and making use again of (2.3a), we have

$$
\sum_{f \in \mathcal{F}} \rho_{f}^{-2 v_{1}^{-}} s_{f}\left|V_{f}\right|^{2} \lesssim \sum_{\tau \in \mathcal{T}}\left\|\rho^{-v_{1}^{-}} V\right\|_{L^{2}(\tau)}^{2}
$$

Combining (5.3) with (5.4) and (5.5) we obtain

$$
\sum_{p \in \mathcal{F}} s_{p}\left|V_{p}\right| \lesssim \sqrt{\sum_{\tau \in \mathcal{T}} \rho_{\tau}^{2 v_{1}^{-}}|\tau|}\left\|\rho^{-v_{1}^{-}} V\right\|_{L^{2}(\Gamma)} \lesssim \sqrt{\sum_{\tau \in \mathcal{T}} \rho_{\tau}^{2 \nu_{1}^{-}}|\tau|}\|V\|_{H^{v_{1}}(\Gamma)},
$$

where the final relation follows from Theorem 3.6 when $v_{1} \leqslant 0$ and trivially otherwise. Using this and an analogous estimate for $\sum_{p \in \mathcal{F}} s_{p}\left|W_{p}\right|$ in (5.2), we obtain the theorem.

\section{REMARK 5.3}

(i) When $\tilde{K}_{f, f^{\prime}}$ is a quadrature approximation of $K_{f, f^{\prime}}$, the value of $\chi$ in Theorem 5.1 is determined by the order of precision of the chosen quadrature rule, the distance between supp $\phi_{f}$ and supp $\phi_{f^{\prime}}$ and the blow-up of the kernel. The rule to be used typically depends on $f, f^{\prime}$ and should be chosen so that the resulting estimates for $r_{\text {stab }}$ and $r_{\text {conv }}$ are suitable for the required application of Lemma 4.2. Typically for kernels $k(\mathbf{x}, \mathbf{y})$ which blow up at $\mathbf{x}=\mathbf{y}$, the order of precision has to be increased when supp $\phi_{p}$ and supp $\phi_{q}$ get closer together (see e.g. Graham et al., 2000a). Special transformation methods can be used to remove the singularity when supp $\phi_{p}$ and supp $\phi_{q}$ intersect (see Hackbusch et al., 1997; Sauter \& Schwab, 2004).

(ii) In Graham et al. (2000a,b) we give a number of different quadrature schemes which can achieve (5.1) in the case when the approximating space is $\mathcal{S}_{1}^{1}(\mathcal{T})$. This analysis can be easily extended to the more general approximating spaces considered here.

A general theory of quadrature approximation of Galerkin methods follows by combining Lemma 4.2 and Theorem 5.1.

COROLlaRY 5.4 Under the conditions of Theorem 5.1, we have

$$
r_{\text {stab }} \lesssim h^{\chi+1}\left\{\sum_{\tau \in \mathcal{T}} \rho_{\tau}^{2 \mu^{-}+1} h_{\tau}\right\}, \quad r_{\text {conv }} \lesssim h^{\chi+1} \sqrt{\sum_{\tau \in \mathcal{T}} \rho_{\tau}^{2 \mu^{-}+1} h_{\tau}} \sqrt{\sum_{\tau \in \mathcal{T}} \rho_{\tau}^{2 v^{-}+1} h_{\tau}} .
$$


Proof. The proof is achieved by a trivial application of Theorem 5.1, with $\nu_{1}=\mu=v_{2}$ for $r_{\text {stab }}$ and $\nu_{1}=\nu, \nu_{2}=\mu$ for $r_{\text {conv }}$, and noting that $|\tau| \sim \rho_{\tau} h_{\tau}$.

When the energy space of the Galerkin method is $H^{\mu}$, for $\mu \geqslant-1 / 2$, we have $2 v^{-}+1 \geqslant 2 \mu^{-}+1 \geqslant$ 0 , and no negative exponent appears in the estimates in Corollary 5.4. Hence the degeneracy has no effect on the stability and consistency estimates. This holds for all the standard boundary integral equations for second-order elliptic PDEs. In particular, for the three standard integral equations given by $(4.4 \mathrm{a}-\mathrm{c})$, we obtain Corollary 5.5, the proof of which follows directly from Corollary 5.4.

COROLlaRY 5.5 For the single-layer potential, we have $\mu=-1 / 2$ and

$$
r_{\text {stab }} \lesssim h^{\chi+1}\left\{\sum_{\tau \in \mathcal{T}} h_{\tau}\right\} \leqslant h^{\chi+2}\{\# \mathcal{T}\},
$$

where $\# \mathcal{T}$ denotes the number of elements in $\mathcal{T}$. With the regularity assumption $\delta=1 / 2$ we may set $v=0$ to obtain

$$
r_{\text {conv }} \lesssim h^{\chi+1}\left\{\sum_{\tau \in \mathcal{T}} h_{\tau}\right\}^{1 / 2}\left\{\sum_{\tau \in \mathcal{T}}|\tau|\right\}^{1 / 2} \lesssim h^{\chi+3 / 2}\{\# \mathcal{T}\}^{1 / 2}
$$

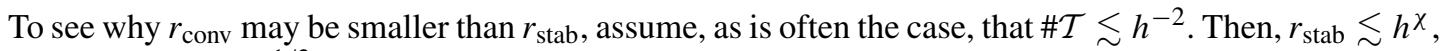
while $r_{\text {conv }} \lesssim h^{\chi+1 / 2}$ and we see the gain of using different quantities for measuring the stability and consistency.

For the double-layer potential and hypersingular operator, we choose $\delta=0$ and $v=\mu$ to obtain in this case

$$
r_{\text {stab }}=r_{\text {conv }} \lesssim h^{\chi+1} \sum_{\tau \in \mathcal{T}}|\tau| \lesssim h^{\chi+1}
$$

\section{Galerkin method with panel clustering}

The panel clustering algorithm provides an alternative representation of the finite-dimensional Galerkin operator described in Section 4, so that multiplication of any vector by the corresponding matrix representation has complexity $\mathcal{O}\left(N \log ^{\kappa} N\right)$, for some (small) $\kappa \sim 4$, where $N(=\# \mathcal{F})$ is the number of degrees of freedom. This should be compared with the $N^{2}$ complexity required for multiplication by the exact matrix. Approximations of this sort are at the heart of many fast methods for dense systems. As well as providing a fast multiplication, the approximation needs also to be sufficiently accurate and, so far, this has only been shown for quasi-uniform meshes. The purpose of this section is to extend the error analysis to (possibly) degenerate meshes. Our results show that the panel clustering approximation satisfies stability and consistency estimates which are independent of mesh degeneracy.

First, we will analyse standard formulations of integral operators in a unified setting. In the final section we consider a special formulation of the hypersingular operator.

\subsection{Panel clustering in the general case}

To obtain this result we need to introduce the following concepts. (For a more complete introduction, see Hackbusch \& Sauter, 1993; Hackbusch et al., 1997, 2000; Sauter, 2000; Börm et al., 2002; Börm \& Hackbusch, 2002.) 
Definition 6.1 (Cluster tree) A cluster tree $\mathbb{T}$ is a tree ${ }^{\dagger}$ whose vertices (called 'clusters') consist of unions $\sigma=\cup\left\{\bar{\tau}: \quad \tau \in \mathcal{T}^{\prime}\right\}$ for certain subsets $\mathcal{T}^{\prime} \subset \mathcal{T}$. These are required to satisfy the following properties:

(i) $\Gamma=\cup_{\tau \in \mathcal{T}} \bar{\tau}$ is the root of $\mathbb{T}$.

(ii) $\mathcal{L}(\mathbb{T})=\mathcal{T}$, where $\mathcal{L}(\mathbb{T})$ denotes the set of leaves of $\mathbb{T}$.

(iii) If $\sigma \in \mathbb{T} \backslash \mathcal{L}(\mathbb{T})$, there is an associated set of vertices of $\mathbb{T}$ (denoted sons $(\sigma)$ ) which satisfies:

(a) $\sigma=\cup\left\{\sigma^{\prime}: \sigma^{\prime} \in \operatorname{sons}(\sigma)\right\}$;

(b) If $\sigma^{\prime}, \sigma^{\prime \prime} \in \operatorname{sons}(\sigma)$ and $\sigma^{\prime} \neq \sigma^{\prime \prime}$, then $\sigma^{\prime}, \sigma^{\prime \prime}$ intersect at most by their boundaries.

There are standard procedures for constructing cluster trees (see for example Börm et al., 2002, Example 2.1). Once $\mathbb{T}$ has been constructed, a second tree, $\mathbb{T}_{2}$, whose vertices are pairs of clusters, may be uniquely defined by the following prescription.

\section{DEFINITION 6.2}

(i) $(\Gamma, \Gamma) \in \mathbb{T}_{2}$ is the root of $\mathbb{T}_{2}$,

(ii) For $b=\left(\sigma^{\prime}, \sigma^{\prime \prime}\right) \in \mathbb{T}_{2}$, the set of sons is defined as follows:

$$
\operatorname{sons}(b):= \begin{cases}\operatorname{sons}\left(\sigma^{\prime}\right) \times \text { sons }\left(\sigma^{\prime \prime}\right) & \text { if } \sigma^{\prime}, \sigma^{\prime \prime} \in \mathbb{T} \backslash \mathcal{L}(\mathbb{T}), \\ \left\{\sigma^{\prime}\right\} \times \text { sons }\left(\sigma^{\prime \prime}\right) & \text { if } b \in \mathcal{L}(\mathbb{T}) \times \mathbb{T} \backslash \mathcal{L}(\mathbb{T}), \\ \operatorname{sons}\left\{\sigma^{\prime}\right\} \times\left\{\sigma^{\prime \prime}\right\} & \text { if } b \in \mathbb{T} \backslash \mathcal{L}(\mathbb{T}) \times \mathcal{L}(\mathbb{T}), \\ \emptyset & \text { if } b \in \mathcal{L}(\mathbb{T}) \times \mathcal{L}(\mathbb{T})\end{cases}
$$

The key point in the panel clustering algorithm is to select pairs of clusters $\left(\sigma^{\prime}, \sigma^{\prime \prime}\right) \in \mathbb{T}_{2}$ and to approximate the corresponding integrals by replacing the kernel $k$ in (4.14) with some suitable separable expansion. This cannot be done on all pairs of clusters, but only on pairs which are sufficiently far apart relative to their diameters. This leads to the following definition of an admissible pair of clusters:.

Definition 6.3 (Admissible pair) For $\eta>0$, a pair $\left(\sigma^{\prime}, \sigma^{\prime \prime}\right) \in \mathbb{T}_{2}$ is called $\eta$-admissible if

$$
\eta \operatorname{dist}\left(\sigma^{\prime}, \sigma^{\prime \prime}\right) \geqslant \max \left\{\operatorname{diam} \sigma^{\prime}, \operatorname{diam} \sigma^{\prime \prime}\right\} \text {. }
$$

Using the concept of admissibility, the integration domain $\Gamma \times \Gamma$ in (4.14) is split into a near field and a far field, characterized by the subsets $P_{\text {far }}$ ('far field') and $P_{\text {near }}$ ('near field') of $\mathbb{T}_{2}$, defined as follows.

First set $P_{\text {near }}=\emptyset=P_{\text {far }}$, and then initiate a call $\operatorname{divide}(\Gamma, \Gamma)$ to the following recursive procedure:

$$
\begin{aligned}
& \text { procedure divide }\left(\sigma^{\prime}, \sigma^{\prime \prime}\right) \text {; } \\
& \text { begin if }\left(\sigma^{\prime}, \sigma^{\prime \prime}\right) \text { is } \eta \text {-admissible then } P_{\text {far }}:=P_{\text {far }} \cup\left\{\left(\sigma^{\prime}, \sigma^{\prime \prime}\right)\right\} \\
& \text { else if }\left(\sigma^{\prime}, \sigma^{\prime \prime}\right) \text { is a leaf then } P_{\text {near }}:=P_{\text {near }} \cup\left\{\left(\sigma^{\prime}, \sigma^{\prime \prime}\right)\right\} \\
& \text { else for all }\left(c^{\prime}, c^{\prime \prime}\right) \in \operatorname{sons}\left(\sigma^{\prime}, \sigma^{\prime \prime}\right) \text { do divide }\left(c^{\prime}, c^{\prime \prime}\right)
\end{aligned}
$$

end;

As a result of this call, $P:=P_{\text {near }} \cup P_{\text {far }}$ describes a non-overlapping covering of $\Gamma \times \Gamma$ in the sense that $\cup\left\{\sigma^{\prime} \times \sigma^{\prime \prime}:\left(\sigma^{\prime}, \sigma^{\prime \prime}\right) \in P\right\}=\Gamma \times \Gamma$ and all contributions $\sigma^{\prime} \times \sigma^{\prime \prime}$ in this union intersect at most by their boundaries.

\footnotetext{
$\dagger$ Usually a tree is a graph $(V, E)$ with vertices $V$ and edges $E$ having a certain structure. Here the structure will be given by the sons of the vertices (defined below), while $V$ is identified with $\mathbb{T}$.
} 
Now we describe how the matrix $K$ is approximated, using this decomposition. For the integration in $P_{\text {far }}$, we approximate the kernel $k(\mathbf{x}, \mathbf{y})$ as follows. Let $b=\left(\sigma^{\prime}, \sigma^{\prime \prime}\right) \in P_{\text {far }}$. For $\mathbf{x} \in \sigma^{\prime}, \mathbf{y} \in \sigma^{\prime \prime}$, we use a separable approximation $k_{b}(\mathbf{x}, \mathbf{y}) \approx k(\mathbf{x}, \mathbf{y})$ of the form

$$
k_{b}(\mathbf{x}, \mathbf{y}):=\sum_{i \in I_{\sigma^{\prime}}, j \in I_{\sigma^{\prime \prime}}} \kappa_{i, j}(b) \Phi_{\sigma^{\prime}}^{(i)}(\mathbf{x}) \Psi_{\sigma^{\prime \prime}}^{(j)}(\mathbf{y})
$$

with appropriate function systems $\left\{\Phi_{\sigma^{\prime}}^{(i)}: i \in I_{\sigma^{\prime}}\right\}$ and $\left\{\Psi_{\sigma^{\prime \prime}}^{(j)}: j \in I_{\sigma^{\prime \prime}}\right\}$ and expansion coefficients $\kappa_{i, j}(b)$.

For kernel functions which are related to linear elliptic PDEs of second order with constant coefficients and when (6.1) is related to a polynomial approximation of degree $l$ in each coordinate direction, one can prove (see Hackbusch \& Nowak, 1989; Sauter \& Schwab, 2004) the exponential convergence estimate

$$
\left|k(\mathbf{x}, \mathbf{y})-k_{b}(\mathbf{x}, \mathbf{y})\right| \leqslant C_{1} \frac{\left(\eta^{\prime}\right)^{l}}{\operatorname{dist}\left(\sigma^{\prime}, \sigma^{\prime \prime}\right)^{s}},
$$

for all $\mathbf{x} \in \sigma^{\prime}, \mathbf{y} \in \sigma^{\prime \prime}$ and $b=\left(\sigma^{\prime}, \sigma^{\prime \prime}\right) \in P_{\text {far }}$, where $\eta^{\prime}=C_{2} \eta$ for some constant $C_{2}$ and $s$ is the blow-up rate of the kernel

$$
|k(\mathbf{x}, \mathbf{y})| \leqslant C_{3}|\mathbf{x}-\mathbf{y}|^{-s}, \quad \mathbf{x}, \mathbf{y} \in \Gamma, \mathbf{x} \neq \mathbf{y} .
$$

Note that the constants $C_{1}$ and $C_{2}$ are independent of $l$ while the cardinality of the index sets $I_{\sigma^{\prime}}, I_{\sigma^{\prime \prime}}$ depends on $l$. In the following, we assume that (6.2) holds.

The panel clustering approximation of the bilinear form $a$ in (4.2) acting on the finite-dimensional space $\mathcal{S}_{i}^{m}(\mathcal{T}) \times \mathcal{S}_{i}^{m}(\mathcal{T})$ is given by

$$
\tilde{a}(V, W)=((\lambda I+\tilde{\mathcal{K}}) V, W), \text { with } \tilde{\mathcal{K}} V(\mathbf{x})=\int_{\Gamma} \tilde{k}(\mathbf{x}, \mathbf{y}) V(\mathbf{y}) \mathrm{d} y, \text { for } V, W \in \mathcal{S}_{i}^{m}(\mathcal{T}),
$$

and

$$
\tilde{k}(\mathbf{x}, \mathbf{y}):= \begin{cases}k(\mathbf{x}, \mathbf{y}) & \mathbf{x} \in \sigma^{\prime}, \mathbf{y} \in \sigma^{\prime \prime} \text { with } b=\left(\sigma^{\prime}, \sigma^{\prime \prime}\right) \in P_{\text {near }}, \\ k_{b}(\mathbf{x}, \mathbf{y}) & \mathbf{x} \in \sigma^{\prime}, \mathbf{y} \in \sigma^{\prime \prime} \text { with } b=\left(\sigma^{\prime}, \sigma^{\prime \prime}\right) \in P_{\text {far. }} .\end{cases}
$$

Since we are concerned here only with error estimates for this approximation, we do not discuss its implementation, but instead refer readers to Hackbusch \& Sauter (1993) and Hackbusch et al. (1997) for details.

Analogously to Theorem 5.1 we then have the following theorem.

THEOREM 6.4 Suppose we use the panel clustering algorithm described above to obtain an approximate bilinear form $\tilde{a}$. Then, for all $v_{1}, v_{2} \in[-1, k]$ such that $\mathcal{S}_{i}^{m}(\mathcal{T}) \subset H^{\max \left\{v_{1}, v_{2}\right\}}(\Gamma)$,

$$
\frac{|a(V, W)-\tilde{a}(V, W)|}{\|V\|_{H^{v_{1}}(\Gamma)}\|W\|_{H^{v_{2}(\Gamma)}}} \lesssim\left(\eta^{\prime}\right)^{l+s}\{\# \mathcal{T}\} \max _{t, \tau \in \mathcal{T}} \Lambda_{t, \tau}^{s}
$$

uniformly in $V, W \in \mathcal{S}_{i}^{m}(\mathcal{T})$, where

$$
\Lambda_{t, \tau}^{s}:=\max \left\{h_{t}, h_{\tau}\right\}^{1-s}\left\{\rho_{t}^{v_{1}^{-}+1 / 2} \rho_{\tau}^{v_{2}^{-}+1 / 2}\right\} \quad \text { and } \quad v_{i}^{-}:=\min \left\{v_{i}, 0\right\}, i=1,2 .
$$

The asymptotic constant in (6.6) may depend on $m$. 
REMARK 6.5 As in Theorem 5.1 this result extends to all $v_{1}, v_{2} \in[-k, k]$ under the assumption that $\chi_{\tau}$ is affine for each $\tau \in \mathcal{T}$.

Proof. By (6.4), (6.5) and (6.2),

$$
\begin{aligned}
|a(V, W)-\tilde{a}(V, W)| & =\left|\sum_{b=\left(\sigma^{\prime}, \sigma^{\prime \prime}\right) \in P_{\text {far }}} \int_{\sigma^{\prime}} \int_{\sigma^{\prime \prime}} V(\mathbf{x})\left(k(\mathbf{x}, \mathbf{y})-k_{b}(\mathbf{x}, \mathbf{y})\right) W(\mathbf{y}) \mathrm{d} y \mathrm{~d} x\right| \\
& \lesssim \sum_{b=\left(\sigma^{\prime}, \sigma^{\prime \prime}\right) \in P_{\text {far }}} \frac{\left(\eta^{\prime}\right)^{l}}{\operatorname{dist}\left(\sigma^{\prime}, \sigma^{\prime \prime}\right)^{s}} \int_{\sigma^{\prime}}|V| \int_{\sigma^{\prime \prime}}|W| \\
& =\sum_{b=\left(\sigma^{\prime}, \sigma^{\prime \prime}\right) \in P_{\text {far }}} \frac{\left(\eta^{\prime}\right)^{l}}{\operatorname{dist}\left(\sigma^{\prime}, \sigma^{\prime \prime}\right)^{s}} \sum_{\substack{t, \tau \in \mathcal{T} \\
t \subset \sigma^{\prime}, \tau \subset \sigma^{\prime \prime}}} \int_{t}|V| \int_{\tau}|W| .
\end{aligned}
$$

Now if $t, \tau \in \mathcal{T}, t \subset \sigma^{\prime}, \tau \subset \sigma^{\prime \prime}$ and $\left(\sigma^{\prime}, \sigma^{\prime \prime}\right) \in P_{\text {far }}$, then $\left(\sigma^{\prime}, \sigma^{\prime \prime}\right)$ is $\eta$-admissible and we have

$$
\eta \operatorname{dist}(t, \tau) \geqslant \eta \operatorname{dist}\left(\sigma^{\prime}, \sigma^{\prime \prime}\right) \geqslant \max \left\{\operatorname{diam} \sigma^{\prime}, \operatorname{diam} \sigma^{\prime \prime}\right\} \geqslant \max \{\operatorname{diam} t, \operatorname{diam} \tau\},
$$

which shows that such $(t, \tau) \in \mathbb{T}_{2}$ are $\eta$-admissible. Since the procedure divide implies that each such $\eta$-admissible $(t, \tau)$ belongs to a unique far field block $\left(\sigma_{t}^{\prime}, \sigma_{\tau}^{\prime \prime}\right) \in P_{\text {far }}$, we can rewrite (6.7) as

$$
|a(V, W)-\tilde{a}(V, W)| \lesssim \sum_{\substack{t, \tau \in \mathcal{T} \\ \eta-\tau \text { admissible }}} \frac{\left(\eta^{\prime}\right)^{l}}{\operatorname{dist}\left(\sigma_{t}^{\prime}, \sigma_{\tau}^{\prime \prime}\right)^{s}} \int_{t}|V| \int_{\tau}|W| .
$$

Because of the properties of $\left(\sigma_{t}^{\prime}, \sigma_{\tau}^{\prime \prime}\right)$,

$$
\operatorname{dist}\left(\sigma_{t}^{\prime}, \sigma_{\tau}^{\prime \prime}\right) \geqslant \eta^{-1} \max \left\{\operatorname{diam}\left(\sigma_{t}^{\prime}\right), \operatorname{diam}\left(\sigma_{\tau}^{\prime \prime}\right)\right\} \geqslant \eta^{-1} \max \left\{h_{t}, h_{\tau}\right\} \gtrsim\left(\eta^{\prime}\right)^{-1} \max \left\{h_{t}, h_{\tau}\right\},
$$

where the constant of proportionality is independent of $\eta$. Moreover, for any $V \in \mathcal{S}_{i}^{m}(\mathcal{T})$ and any $\tau \in \mathcal{T}$, we have, by Assumption 2.6, for any $v \in \mathbb{R}$,

$$
\int_{\tau}|V| \sim \int_{\tau} \rho_{\tau}^{\nu^{-}}\left|\rho^{-\nu^{-}} V\right| \leqslant \sqrt{|\tau| \rho_{\tau}^{2 \nu^{-}}}\left\|\rho^{-\nu^{-}} V\right\|_{L^{2}(\tau)},
$$

where $v^{-}=\min \{v, 0\}$. Inserting these last two results into (6.9), we obtain

$$
\begin{aligned}
|a(V, W)-\tilde{a}(V, W)| & \\
& \lesssim \sum_{\substack{t, \tau) \\
\eta-\text { admissible }}} \frac{\left(\eta^{\prime}\right)^{l+s}}{\max \left\{h_{t}, h_{\tau}\right\}^{s}} \sqrt{|t| \rho_{t}^{2 v_{1}^{-}}|\tau| \rho_{\tau}^{2 v_{2}^{-}}}\left\|\rho^{-v_{1}^{-}} V\right\|_{L^{2}(t)}\left\|\rho^{-v_{2}^{-}} W\right\|_{L^{2}(\tau)} \\
& \lesssim\left(\eta^{\prime}\right)^{l+s} \max _{t, \tau \in \mathcal{T}} \frac{\sqrt{|t| \rho_{t}^{2 v_{1}^{-}}|\tau| \rho_{\tau}^{2 v_{2}^{-}}}}{\max \left\{h_{t}, h_{\tau}\right\}^{s}} \sum_{t \in \mathcal{T}}\left\|\rho^{-v_{1}^{-}} V\right\|_{L^{2}(t)} \sum_{\tau \in \mathcal{T}}\left\|\rho^{-v_{2}^{-}} W\right\|_{L^{2}(\tau)} .
\end{aligned}
$$

Now observe that (since here $\Gamma$ is a two-dimensional manifold), for all $t, \tau \in \mathcal{T}$,

$$
\frac{\sqrt{|t| \rho_{t}^{2 v_{1}^{-}}|\tau| \rho_{\tau}^{2 v_{2}^{-}}}}{\max \left\{h_{t}, h_{\tau}\right\}^{s}} \sim \frac{\sqrt{h_{t} h_{\tau}}}{\max \left\{h_{t}, h_{\tau}\right\}^{s}}\left\{\rho_{t}^{v_{1}^{-}+1 / 2} \rho_{\tau}^{v_{2}^{-}+1 / 2}\right\} \leqslant \max \left\{h_{t}, h_{\tau}\right\}^{1-s}\left\{\rho_{t}^{v_{1}^{-}+1 / 2} \rho_{\tau}^{v_{2}^{-}+1 / 2}\right\} .
$$


The result follows from (6.11) by first applying the Cauchy-Schwarz inequality and then Theorem 3.6 — when either $v_{1}$ or $v_{2}$ is negative — or a trivial inequality otherwise.

In Theorem 6.4, $l$ and $\eta$ are parameters which control the accuracy of the panel clustering algorithm. (They also control its computational cost.) Using Theorem 6.4 we can see how $l$ and $\eta$ should be chosen in order to ensure the required accuracy in the case of various standard integral equations. The results are in the following corollary.

COROLLARY 6.6 Under the assumptions of Theorem 6.4, we have

(i) For the single-layer potential: $\quad r_{\text {stab }} \lesssim\left(\eta^{\prime}\right)^{l+1}\{\# \mathcal{T}\}$,

(ii) For the double-layer potential: $\quad r_{\text {stab }} \lesssim\left(\eta^{\prime}\right)^{l+2}\{\# \mathcal{T}\}$,

(iii) For the hypersingular operator: $\quad r_{\text {stab }} \lesssim\left(\eta^{\prime}\right)^{l+3}\{\# \mathcal{T}\} \max _{\tau \in \mathcal{T}}\left\{h_{\tau}^{-1}\right\}$.

According to Remark 4.3, the choice $v=\mu$ and $\delta=0$ is optimal for the double-layer potential and the hypersingular operator. In this case $r_{\text {stab }}=r_{\text {conv }}$.

If we assume for the single-layer potential $L^{2}$-regularity, i.e. $\delta=1 / 2$ in (4.18) we may choose $v=0$ to obtain

$$
r_{\text {conv }} \lesssim h^{1 / 2}\left(\eta^{\prime}\right)^{l+1}\{\# \mathcal{T}\}
$$

Proof. Putting $v_{1}=\mu=v_{2}$ in the result of Theorem 6.4, we obtain

$$
r_{\text {stab }} \lesssim\left(\eta^{\prime}\right)^{l+s}\{\# \mathcal{T}\} \max _{t, \tau \in \mathcal{T}}\left\{\max \left\{h_{t}, h_{\tau}\right\}^{1-s}\left\{\rho_{t} \rho_{\tau}\right\}^{\mu^{-}+1 / 2}\right\}
$$

where $\mu^{-}=\min \{\mu, 0\}$.

The estimate (i) follows easily since, for the single-layer potential, $s=1$ and $\mu=-1 / 2$.

The estimate for $r_{\text {conv }}$ in the case $\delta=1 / 2$ and $v=0$ follows from (6.6) with $s=1, v_{1}=0, v_{2}=$ $-1 / 2$ :

$$
\begin{aligned}
r_{\text {conv }} & \lesssim\left(\eta^{\prime}\right)^{l+s}\{\# \mathcal{T}\} \max _{t, \tau \in \mathcal{T}}\left\{\rho_{t}^{v_{1}^{-}+1 / 2} \rho_{\tau}^{v_{2}^{-}+1 / 2} \max \left\{h_{t}, h_{\tau}\right\}^{1-s}\right\} \\
& =\left(\eta^{\prime}\right)^{l+1}\{\# \mathcal{T}\}\left(\max _{t \in \mathcal{T}} \rho_{t}^{1 / 2}\right) \lesssim h^{1 / 2}\left(\eta^{\prime}\right)^{l+1}\{\# \mathcal{T}\} .
\end{aligned}
$$

For the double-layer potential (on a polyhedron) we have $s=2$ and $\mu=0$. Then (6.12) leads to

$$
r_{\text {stab }} \lesssim\left(\eta^{\prime}\right)^{l+2}\{\# \mathcal{T}\} \max _{t, \tau \in \mathcal{T}}\left\{\sqrt{\rho_{t} \rho_{\tau}} \max \left\{h_{t}, h_{\tau}\right\}^{-1}\right\} .
$$

Since $\max \left\{h_{t}, h_{\tau}\right\}^{-1} \leqslant\left\{h_{t} h_{\tau}\right\}^{-1 / 2}$, and since $\rho_{\tau} \leqslant h_{\tau}$, for all $\tau \in \mathcal{T}$, the result (ii) follows.

In the hypersingular case $s=3$ and $\mu=1 / 2$ and (6.12) readily yields

$$
r_{\text {stab }} \lesssim\left(\eta^{\prime}\right)^{l+3}\{\# \mathcal{T}\} \max _{t, \tau \in \mathcal{T}} \max \left\{h_{t}, h_{\tau}\right\}^{-1}
$$

which yields the required result.

EXAMPLE 6.7 Let $\Gamma$ be the surface of the unit cube $[0,1]^{3}$ and consider problem (4.2) in the special case (4.4a): Find $u \in H^{-1 / 2}(\Gamma)$ such that

$$
\int_{\Gamma} \frac{u(\mathbf{y}) v(\mathbf{x})}{4 \pi|\mathbf{x}-\mathbf{y}|} \mathrm{d} x \mathrm{~d} y=\int_{\Gamma} g(\mathbf{x}) v(\mathbf{x}) \mathrm{d} x \quad \forall v \in H^{-1 / 2}(\Gamma),
$$


for a given (say smooth) function $g$ on $\Gamma$. It is known that the Galerkin discretization in $\mathcal{S}_{0}^{1}(\mathcal{T})$ on the mesh is obtained by applying the mesh in Example 2.7 on each face of $\Gamma$; then we obtain a solution which satisfies the error estimate (see Petersdorff, 1989, Sauter \& Schwab, 2004)

$$
\|u-U\|_{H^{-1 / 2}(\Gamma)} \leqslant C N^{-\min \{g-\varepsilon, 5\} / 4},
$$

where $0<\varepsilon<1$ is arbitrary but fixed, $N=\operatorname{dim} \mathcal{S}_{0}^{1}(\mathcal{T})$, and $g$ denotes the grading exponent. The choice $g>5$ leads to the optimal convergence rate of $N^{-5 / 4}$.

For this problem, Corollary 6.6(i) tells us that provided $\left(\eta^{\prime}\right)^{l+1} N \lesssim N^{-5 / 4}$, then optimal convergence will follow. Hence, by the trivial estimate (4.25) the Galerkin solution converges with optimal rate if the expansion order for the panel clustering algorithm is chosen according to

$$
l=\left\lceil\frac{9}{4} \frac{\log N}{\left|\log \eta^{\prime}\right|}\right\rceil .
$$

The estimates given in Corollary 6.6 for the single- and double-layer potentials are clearly unaffected by any mesh degeneracy. However in the case of the hypersingular operator, a negative power of the minimum diameter occurs. This is not a severe deficiency, but nevertheless it can be removed if we reformulate the hypersingular equation using the concept of partial integration. This we describe in the following final section.

\subsection{Hypersingular operator with partial integration}

The integral of the kernel of the hypersingular operator in (4.4c) does not exist as an improper integral and has to be defined as a finite part integral. Various regularization methods for hypersingular integrals exist in the literature and we choose here the method of partial integration (see Nédélec, 2001). As explained in Remark 4.1, we restrict for the hypersingular operator (see (4.4c)) to the case $\omega>0$ allowing the choice $\mathcal{V}^{1 / 2}=H^{1 / 2}(\Gamma)$, while the case $\omega=0$ can be treated similarly, but with some more technicalities.

The bilinear form $a: H^{1 / 2}(\Gamma) \times H^{1 / 2}(\Gamma) \rightarrow \mathbb{R}$ which is associated with the hypersingular operator can be written in the form (see Nédélec, 2001; Sauter \& Schwab, 2004)

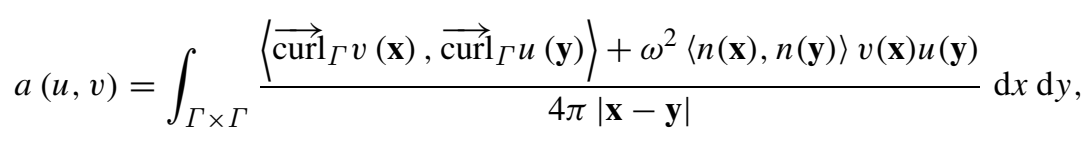

where the tangential rotation $\overrightarrow{c u r l}_{\Gamma}$ is defined as follows (see Nédélec, 2001). For functions $u \in$ $H^{1 / 2}(\Gamma)$ and surface vector fields having componentwise differentiable extensions $\tilde{u}$ and $\tilde{v}$, respectively, in $H^{1}(\mathcal{U})$, where $\mathcal{U}$ is some three-dimensional neighbourhood of $\Gamma$, we define the tangential gradient $\nabla_{\Gamma} u$ as the restriction of the Euclidean gradient to the surface $\Gamma$

$$
\nabla_{\Gamma} u:=\left.(\nabla \tilde{u})\right|_{\Gamma} .
$$

This enables us to introduce the tangential rotation of $u$ as

$$
\left.\overrightarrow{\operatorname{cur}}\right|_{\Gamma} u:=-n \times \nabla_{\Gamma} u .
$$


Since the energy space for $(6.13)$ is $H^{1 / 2}(\Gamma)$ we must use continuous piecewise polynomials $\mathcal{S}_{1}^{m}(\mathcal{T})$ for its discretization. To analyse the effect of the approximation of the bilinear form $a(\cdot, \cdot)$ by the panel clustering algorithm, the estimate of the quantities

$$
r_{\text {stab }}:=\frac{|a(V, W)-\tilde{a}(V, W)|}{\|V\|_{H^{1 / 2}(\Gamma)}\|W\|_{H^{1 / 2}(\Gamma)}} \quad \text { and } \quad r_{\text {conv }}:=\sup _{V, W \in \mathcal{S}_{i}^{m}(\mathcal{T}) \backslash\{0\}} \frac{|a(V, W)-\tilde{a}(V, W)|}{\|V\|_{H^{\nu}(\Gamma)}\|W\|_{H^{1 / 2}(\Gamma)}}
$$

for all $V, W \in \mathcal{S}_{1}^{m}(\mathcal{T}) \backslash\{0\}$ plays the essential role. The parameter $v$ must satisfy (4.20) with $\mu=1 / 2$.

We split the bilinear form $a(\cdot, \cdot)=a_{1}(\cdot, \cdot)+a_{2}(\cdot, \cdot)$ in $(6.13)$ according to the sum in the numerator of the integrand.

To estimate the perturbation in $a_{2}$, we can employ (6.12) with $s=1$ and $\mu=1 / 2$ to obtain, for all $v \geqslant 1 / 2$,

$$
r_{\text {conv }}^{(2)} \leqslant r_{\text {stab }}^{(2)} \lesssim\left(\eta^{\prime}\right)^{l+1}\{\# \mathcal{T}\} h
$$

where the indices (2) in the left-hand side of (6.15) correspond to the bilinear form $a_{2}(\cdot, \cdot)$.

Next, we turn to the estimate for the perturbation error in $a_{1}(\cdot, \cdot)$. For simplicity, we assume that the piecewise smooth Lipschitz surface $\Gamma$ is actually the surface of a polyhedron

$$
\Gamma=\bigcup_{i=1}^{q} \Gamma_{i} \quad \text { where every } \Gamma_{i}, 1 \leqslant i \leqslant q \text {, is planar. }
$$

As a consequence, the normal $n$ is constant on every panel $\tau \in \mathcal{T}$ and

$$
\overrightarrow{\operatorname{curl}_{\Gamma}} \mathcal{S}_{1}^{m}(\mathcal{T}):=\left\{\overrightarrow{\operatorname{curl}_{\Gamma}} u: u \in \mathcal{S}_{1}^{m}(\mathcal{T})\right\} \subset\left(\mathcal{S}_{0}^{m-1}(\mathcal{T})\right)^{3}
$$

THEOREM 6.8 Let $r_{\text {stab }}^{(1)}, r_{\text {conv }}^{(1)}$ be defined as in (6.14) with respect to the bilinear form $a_{1}(\cdot, \cdot)$ as in (6.13) and denote its panel clustering approximation by $\tilde{a}_{1}(\cdot, \cdot)$. Assume that $\Gamma$ satisfies (6.16). Then the stability estimate

$$
r_{\text {stab }}^{(1)} \lesssim\left(\eta^{\prime}\right)^{l+1}\{\# \mathcal{T}\}
$$

holds uniformly in $V, W \in \mathcal{S}_{1}^{m}(\mathcal{T}) \backslash\{0\}$. The choice $v=1 / 2$ leads to $r_{\text {conv }}=r_{\text {stab }}$.

Assume that the continuous problem has regularity $\delta=1 / 2$. In this case, we have

$$
r_{\text {conv }}^{(1)} \lesssim h^{1 / 2}\left(\eta^{\prime}\right)^{l+1}(\# \mathcal{T})
$$

Proof. It is well known that the bilinear form $a(\cdot, \cdot)$ is elliptic and continuous in $H^{1 / 2}(\Gamma)$ (see Nédélec, 2001; Sauter \& Schwab, 2004). In view of the inclusion in (6.17) we may use the inverse inequality from Theorem 3.4 for $s=1, \alpha=1 / 2$ and interpolate with the trivial identity $\left\|\rho^{1 / 2} V\right\|_{H^{1}(\Omega)}=\left\|\rho^{1 / 2} V\right\|_{H^{1}(\Omega)}$ to obtain

$$
\left\|\rho^{1 / 2} \overrightarrow{\operatorname{curl}} \Gamma_{\left(L^{2}(\Gamma)\right)^{3}} \leqslant\right\| \rho^{1 / 2} V\left\|_{H^{1}(\Gamma)} \lesssim\right\| V \|_{H^{1 / 2}(\Gamma)} \quad \text { for all } V \in \mathcal{S}_{1}^{m}
$$

Hence,

$$
\frac{\left|a_{1}(V, W)-\tilde{a}_{1}(V, W)\right|}{\|V\|_{H^{1 / 2}(\Gamma)}\|W\|_{H^{1 / 2}(\Gamma)}} \lesssim \frac{\left|a_{1}(V, W)-\tilde{a}_{1}(V, W)\right|}{\left\|\rho^{1 / 2} V^{c}\right\|_{L^{2}(\Gamma)}\left\|\rho^{1 / 2} W^{c}\right\|_{L^{2}(\Gamma)}},
$$


where $V^{c}:=\overrightarrow{\operatorname{curl}_{\Gamma}} V$ and $W^{c}:=\overrightarrow{\operatorname{curl}_{\Gamma}} W$. Now, by repeating the steps in the proof of Theorem 6.4, we obtain the estimate for $a_{1}-\tilde{a}_{1}$ :

$$
\begin{aligned}
\left|a_{1}(V, W)-\tilde{a}_{1}(V, W)\right| & =\sum_{b=\left(\sigma^{\prime}, \sigma^{\prime \prime}\right) \in P_{\mathrm{far}}} \int_{\sigma^{\prime}} \int_{\sigma^{\prime \prime}}\left\langle V^{c}(\mathbf{x}), W^{c}(\mathbf{y})\right\rangle\left|k(\mathbf{x}, \mathbf{y})-k_{b}(\mathbf{x}, \mathbf{y})\right| \mathrm{d} x \mathrm{~d} y \\
& \lesssim \sum_{\substack{t, \tau \in \mathcal{T} \\
(t, \tau)}-\text { admissible }} \frac{\left(\eta^{\prime}\right)^{l}}{\operatorname{dist}\left(\sigma_{t}^{\prime}, \sigma_{\tau}^{\prime \prime}\right)} \int_{t}\left|V^{c}\right| \int_{\tau}\left|W^{c}\right|
\end{aligned}
$$

Moreover, for any $V^{c} \in \mathcal{S}_{0}^{m-1}(\mathcal{T})$ and any $\tau \in \mathcal{T}$, we have, by Assumption 2.6,

$$
\int_{t}\left|V^{c}\right| \sim \int_{t} \rho_{t}^{-1 / 2}\left|\rho^{1 / 2} V^{c}\right| \leqslant\left\{|t| \rho_{t}^{-1}\right\}^{1 / 2}\left\|\rho^{1 / 2} V^{c}\right\|_{L^{2}(t)} \lesssim h_{t}^{1 / 2}\left\|\rho^{1 / 2} V^{c}\right\|_{L^{2}(t)} .
$$

Inserting this and (6.10) into (6.19), we obtain

$$
\left|a_{1}(V, W)-\tilde{a}_{1}(V, W)\right| \lesssim\left(\eta^{\prime}\right)^{l+1}\left[\max _{\tau, t \in \mathcal{T}}\left\{\frac{\left(h_{t} h_{\tau}\right)^{1 / 2}}{\max \left\{h_{t}, h_{\tau}\right\}}\right\}\right] \sum_{t \in \mathcal{T}}\left\|\rho^{1 / 2} V^{c}\right\|_{L^{2}(t)} \sum_{\tau \in \mathcal{T}}\left\|\rho^{1 / 2} W^{c}\right\|_{L^{2}(\tau)}
$$

Since $\sqrt{h_{t} h_{\tau}} \leqslant \max \left\{h_{t}, h_{\tau}\right\}$, the term in square brackets in (6.20) is bounded from above by 1 and we obtain by the Cauchy-Schwarz inequality the final estimate

$$
\left|a_{1}(V, W)-\tilde{a}_{1}(V, W)\right| \lesssim\left(\eta^{\prime}\right)^{l+1}(\# \mathcal{T})\left\|\rho^{1 / 2} V^{c}\right\|_{L^{2}(\Gamma)}\left\|\rho^{1 / 2} W^{c}\right\|_{L^{2}(\Gamma)} .
$$

Combining this with (6.18) yields the estimate for $r_{\text {stab }}^{(1)}$.

Assume that the problem has regularity $\delta=1 / 2$. Repeating the proof for $r_{\text {stab }}^{(1)}$ but applying the inverse estimate only for the function $W$, we obtain

$$
\left|a_{1}(V, W)-\tilde{a}_{1}(V, W)\right| \lesssim h^{1 / 2}\left(\eta^{\prime}\right)^{l+1}(\# \mathcal{T})\|V\|_{H^{1}(\Gamma)}\|W\|_{H^{1 / 2}(\Gamma)} .
$$

REMARK 6.9 Theorem 6.8 along with estimate (6.15) shows that the negative power of $h$ in Corollary 6.6(iii) can be avoided by applying the panel clustering algorithm to the kernel in (6.13) and not to the hypersingular kernel function in its original form (4.4c). In addition, we gain an additional factor $h^{1 / 2}$ in the error estimate by employing the regularity of the solution.

\section{Acknowledgements}

IGG would like to thank the Max-Planck-Institut für Mathematik in den Naturwissenschaften, Leipzig and Universität Zürich for financial support. SAS was supported by the Swiss National Science Foundation, Grant 21-61764.00. 


\section{REFERENCES}

BÖRM, S. \& HACKBUSCH, W. (2002) Data-sparse approximation by adaptive $\mathcal{H}^{2}$-matrices. Computing, 69, $1-35$.

BÖrm, S., Grasedyck, L. \& Hackbusch, W. (2002) An introduction to hierarchical matrices. Math. Bohem., 127, 229-241.

Ciarlet, P. G. (1978) The Finite Element Method for Elliptic Problems. Amsterdam: North-Holland.

Dahmen, W., Faermann, B., Graham, I. G., Hackbusch, W. \& Sauter, S. A. (2004) Inverse inequalities on non-quasiuniform meshes and application to the mortar element method. Math. Comp., 73, 1107-1138.

ELSCHNER, J. (1992) The double-layer potential operator over polyhedral domains II: Spline Galerkin methods. Math. Methods Appl. Sci., 15, 23-37.

Ervin, V. J., Stephan, E. P. \& Abou El-Seoud, A. (1990) An improved boundary element method for the charge density of a thin electrified plate in $\mathbb{R}^{3}$. Math. Methods Appl. Sci., 13, 291-303.

FAERMANN, B. (2002) Localization of the Aronszajn-Slobodeckij norm and application to adaptive boundary element methods. Part II. The three-dimensional case. Numer. Math., 92, 467-499.

Graham, I. G., Hackbusch, W. \& SAuter, S. A. (1999) Hybrid Galerkin boundary elements on degenerate meshes. Mathematical Aspects of Boundary Element Methods. (M. Bonnet, A.-M. Sändig \& W. L. Wendland, eds). Boca Raton, FL: CRC Press.

Graham, I. G., Hackbusch, W. \& SAuter, S. A. (2000a) Discrete boundary element methods on general meshes in 3D. Numer. Math., 86, 103-137.

Graham, I. G., HACKBUSCH, W. \& SAUTER, S. A. (2000b) Hybrid Galerkin boundary element methods. Numer. Math., 86, 139-172.

Graham, I. G., Hackbusch, W. \& SAUter, S. A. Panel-clustering for stretched elements. in preparation.

HaCkBusch, W. (1992) Elliptic Differential Equations. Theory and Numerical Treatment SCM, Vol. 18. Berlin: Springer.

HACKBUSCH, W. \& NOWAK, Z. (1989) On the fast matrix multiplication in the boundary element method by panel clustering. Numer. Math., 54, 463-491.

Hackbusch, W. \& SAUter, S. A. (1993) On the efficient use of the Galerkin method to solve Fredholm integral equations. Appl. Math., 38, 301-322.

Hackbusch, W., LAGE, C. \& SAUter, S. A. (1997) On the efficient realization of sparse matrix techniques for integral equations with focus on panel clustering, cubature and software design aspects. Boundary Element Topics. (W. L. Wendland, ed.). Berlin: Springer, pp. 51-76.

Hackbusch, W., Khoromskiu, B. \& SAUter, S. A. (2000) On $\mathcal{H}^{2}$ matrices. Lectures on Applied Mathematics. (H.-J. Bungartz, R. H. W. Hoppe \& C. Zenger, eds). Heidelberg: Springer, pp. 9-30.

MCLean, W. (2000) Strongly Elliptic Systems and Boundary Integral Equations. Cambridge: Cambridge University Press.

NÉDÉLEC, J. C. (2001) Acoustic and Electromagnetic Equations. New York: Springer.

NOBLE, B. (1961) The numerical solution of the singular integral equation for the charge distribution on a flat rectangular lamina. Proc. Symp. on the Numerical Solution of Differential and Integral Equations. Basel: Birkhauser, pp. 530-543.

PETERSDORFF, T. V. (1989) Elasticity problems in polyhedra-singularities and approximation with boundary elements. Ph.D. Dissertation, TH Darmstadt.

SAUTER, S. A. (1997) The panel custering method in 3-d BEM. Wave Propagation in Complex Media, IMAVolumes in Mathematics and its Applications, Vol. 96. (G. Papanicolaou, ed.). Heidelberg: Springer, pp. 199224.

SAUTER, S. A. (2000) Variable order panel clustering. Computing, 64, 223-261.

SAuter, S. A. \& Schwab, C. (1997) Quadrature for $h p$-Galerkin BEM in 3D. Numer. Math., 78, 211-258.

SAUter, S. A. \& SCHWAB, C. (2004) Randelementmethoden. Leipzig: Teubner. 